Original Research Paper

\title{
Trajectory Optimization for First Human Asteroid Exploration Mission
}

\author{
${ }^{1}$ Atiksha Sharma and ${ }^{2}$ Santosh Kosambe \\ ${ }^{1}$ Independent Researcher, Nagpur, India \\ ${ }^{2}$ Independent Researcher, Pune, India
}

\section{Article history}

Received: 06-03-2020

Revised: 21-04-2020

Accepted: 27-07-2020

Corresponding Author:

Atiksha Sharma

Independent Researcher,

Nagpur, India

Email: atiksharma91@gmail.com

\begin{abstract}
After the successful completion of robotic expeditions on Mars, an ultimate goal was set for space fairing nations and agencies around the world to send astronauts to Mars by 2030. Unfortunately, a mission to Mars would last upwards for years meanwhile several asteroids fly close to the Earth. A manned trip to an asteroid would be a valuable stepping stone towards a manned mission to Mars, as the mission time can be reduced to a few months round-trip. In addition to gaining experience with manned travel through deep space, a mission to an asteroid would provide experience overcoming a unique challenge of rendezvousing with a foreign object in microgravity. Since most of the known asteroids that are close enough to Earth to be viable targets are less than $300 \mathrm{~m}$ in diameter, chances are the selected asteroid will not have a significant gravitational field. Near Earth Asteroids (NEAs) have orbits that bring them into close proximity with Earth's orbit making them both a unique hazard to life on Earth and a unique opportunity for science and exploration. A specific case study was therefore undertaken to shortlist such NEAs that are accessible for round-trip human missions using heavy lift launch architecture. A fully parameterized, highly efficient algorithm is developed using MATLAB to accomplish this. Using this algorithm a family of NEA is reviewed, several candidate asteroids are shortlisted for potential exploration and a final selection of asteroid 1999 AO10 is made followed by a specific mission trajectory design and selection to this asteroid, accounting for mission requirements and constraints. The astrodynamic components of a manned mission to NEA 1999 AO10 are also described in this study. Beginning with a launch, we will walk through preliminary trajectory optimization using Lambert's problem and patched conics. Once the ideal trajectory is designed, the study will be extended to include abort scenarios.
\end{abstract}

Keywords: Near Earth Asteroids, 1999 AO10, Trajectory Optimization, Asteroid Mission, Asteroid Exploration, Crewed Exploration

\section{Introduction}

Asteroids are ancient, small metallic objects that orbit around the Sun but are too small to be classified as planets. Large numbers of these ranges enormously in size, from Ceres, the largest object in the asteroid belt known to date to bodies that are about the size of pebbles. Each asteroid is traveling around the Sun fast enough for the orbits not to degrade. If something slows an asteroid, it may fall towards the Sun, Mars, or Jupiter. There are many theories about the formation of asteroids in our solar system. Some scientists believe that the asteroid belt was made when a planet that was there exploded or collided with something else and broke up. Other scientists believe that the material making the asteroids never came together into a planet at all. While some researchers believe that the asteroids are remnants left over from the early formation of our solar system about 4.6 billion years ago when a big cloud of gas and dust collapsed.

The majority of known asteroids in our solar system are orbiting in broadband of 19.4 million miles of Main Asteroid Belt, a doughnut-shaped ring which lies between the orbits of Mars and Jupiter, generally in 
relatively low-eccentricity orbits (Fig. 1). This belt is now estimated to contain 1.1 to 1.9 million asteroids larger than $1 \mathrm{~km}$ in diameter and millions of smaller ones. As of July 2020, the Minor Planet Centre had observed 264.8 million objects in the inner and outer solar system, of which 546,077 had enough information to be given numbered designations. According to the MPC database near about 959,226 asteroids have been discovered so far (MPC, 2020). Most of the undiscovered asteroids are the smaller ones (less than $100 \mathrm{~km}$ across) which are more difficult to detect.

Scientists have discovered various dynamical groups of asteroids orbiting in the inner solar system. Orbits of such asteroids are perturbed by the gravity of other bodies in the solar system and by the Yarkovsky effect. Significant populations include Main Asteroid Belt, Trojans and NEAs. The majority of asteroids are further classified into three categories namely C-type (chondrite), S-type (stony) and M-type (metallic) according to their albedo, composition derived from spectral features in their reflected sunlight and inferred similarities to known meteorite types. Trojans are populations that share an orbit with a larger planet or moon but do not collide with it because they orbit in one of the two Lagrangian points of stability, L4 and L5, which lie $60^{\circ}$ ahead of and behind the Jupiter.

Asteroids that have been nudged by the gravitational attraction of nearby planets into orbits that allow them to pass close to that of Earth's orbit are known as Earth approaching or NEAs. In terms of orbital elements, NEAs are asteroids with a perihelion distance of less than 1.3 AU. Asteroids that cross Earth's orbital path are known as Earth-crossers. As of July 23, 2020, more than
23,121 known NEAs are cataloged in the Center for Near-Earth Object Studies (CNEOS) database and more are being discovered on a continual basis (CNEOS, 2020). It is believed that the total NEA population consists of at least thousands of millions of objects. Most of the NEAs are fragments jarred from the main belt by a combination of asteroid collisions and the gravitational influence of Jupiter. Some NEAs may be the nuclei of the dead, short-period comets. The NEA population appears to be representative of most or all asteroid types found in the main belt. NEAs are divided into four groups according to their perihelion distance (q), aphelion distance (Q) and their semi-major axes (a) as Atira, Aten, Apollo and Amor. Asteroids that cross Mars orbit but do not quite reach the orbit of Earth are known as Amor. Apollo and Aten are asteroids that cross Earth's orbit with a period greater and less than 1 year respectively. Finally, asteroids that have orbits contained entirely within the orbit of the Earth are known as Atira.

The asteroid 1999 AO10 is often identified as a potential candidate for designing missions to Near Earth Objects (NEO). Its orbit is similar to Earth's orbit and it has close approach dates in the mid-2025s, according to JPL's Small-Body Database. Figure 2 shows the orbits of Earth and 1999 AO10 around the Sun. The image was generated with JPL HORIZONS using planetary ephemeris data from JPL's small body browser, propagated to September 19, 2025. In this paper, we have designed the trajectory to asteroid 1999 AO10 which is accessible for the first human exploration mission using the designed algorithm. Also, we have presented the methodology to design the trajectory utilizing heavy-lift launch architecture.

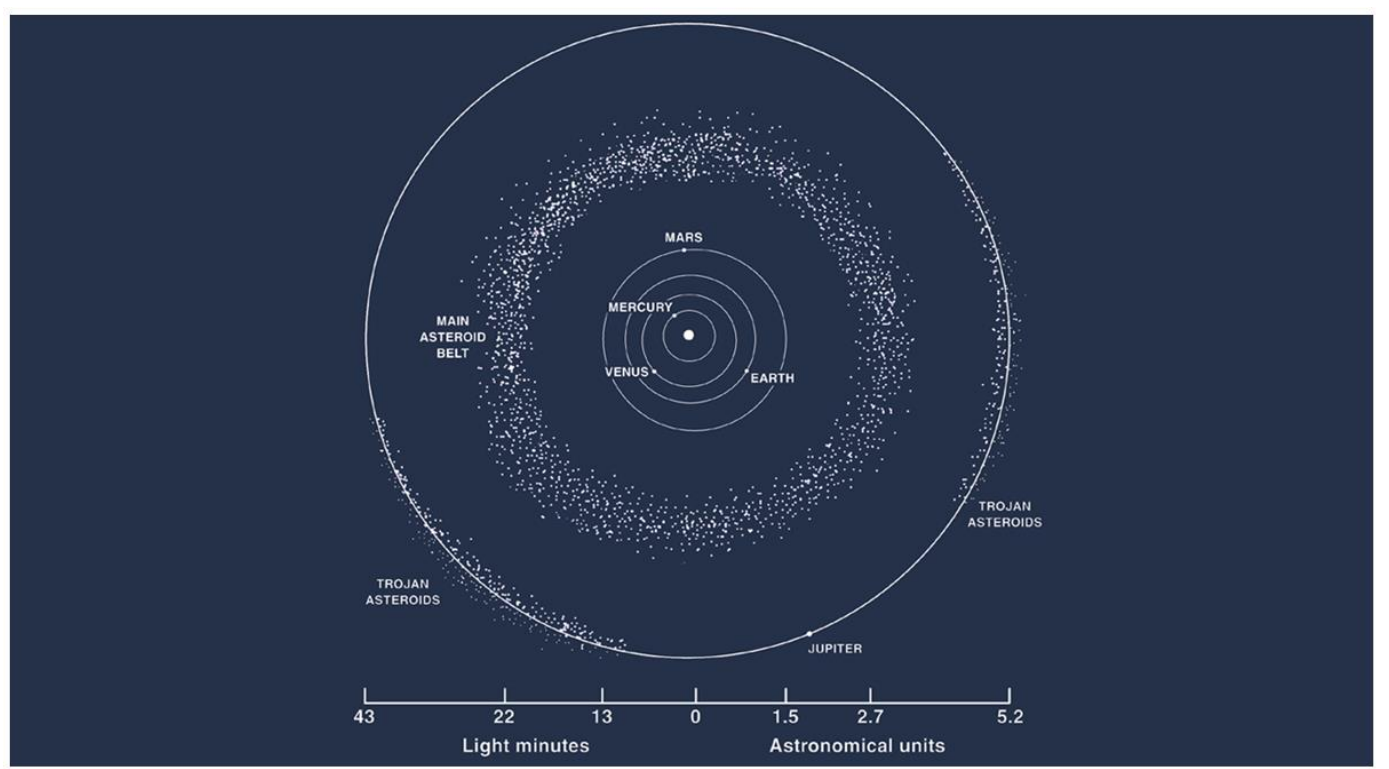

Fig. 1: Distribution of asteroids in orbits around the Sun (Credit - NASA) 


\section{Reasons for NEA Missions}

There are numerous reasons, benefits and rationale to visit NEAs and send both robotic and crewed missions to study them. NEAs are among the vital targets for fundamental solar system science and the collision threat they pose to life on Earth makes it even more imperative that we understand their sizes, compositions, internal structures, spin states and orbits, among other characteristics, so that we may be prepared to act when an NEA on a collision course with Earth is discovered. The close proximity of NEA orbits to Earth's orbit also raises the possibility of short duration round trip missions to them, which would allow us to send humans to visit NEAs. Firstly, a crewed mission to an NEA is less risky compared to a lunar or Mars mission due to the absence of lander to get to NEA. Also, it may be possible to utilize asteroids for their material resources. An NEA mission would also help develop deep-space operational experience. This will be critical for a crewed Mars mission and any other mission aimed at building a human presence in the inner solar system. The further detailed analysis could offer insight into how the solar system was formed. Finally and perhaps most importantly, a crewed NEA mission would serve as a crucial stepping stone to Mars. Besides, having the capability to reach NEA enables deflection missions, if ever necessary.

\section{Solar System Science}

Asteroids are largely unchanged in composition since the early days of our solar system and studying them provides vital insight into our origins. Asteroids such as 2 Pallas and 10 Hygiea, which are both believed to have water in the past, appear to have organic (carbon-based) compounds on them. Today, these asteroids have a more primitive chemical composition than Earth has. They are more similar to the conditions that existed in the solar system's younger years. By studying them, we may learn about how life arose on our own planet. Some conditions may have been conducive to life in the past. Additionally, it is possible that these asteroids may have delivered vast quantities of water to the young Earth and may have also carried the seeds of life itself. Another tantalizing possibility is that besides learning about our own solar system, we can apply this knowledge to understand other star systems and the potential for life elsewhere in the universe. Besides these, asteroids are also interesting from a scientific perspective, because studying them complements our studies of the major planets. Analyzing the differences between the planets and the asteroids is like taking slices of the solar system at different times during its formation. Stray asteroids and asteroid fragments slammed into Earth and the other planets in the past played a major role in altering the geological history of the planets and in the evolution of life on Earth. The materials present in these asteroids represent the building blocks of the planets. Because of the position of the asteroid belt that lies between the rocky inner planet and the gas giants of the outer solar system, the materials found there may hold clues as to why the planets are so diverse today. Scientists do not fully understand how the first life forms arose on Earth from nonliving organic matter and asteroids may help us learn more about this puzzle. Plus, scientists think asteroids that landed on Earth long ago may have deposited some of the building blocks that helped start a life here.

\section{Impact Hazard and Planetary Defense}

NEAs pose a hazard to Earth as they can collide with our planet, sometimes to devastating effect. As of July 23, 2020, 2099 NEAs were classified as Potentially Hazardous Asteroids (PHAs) (CNEOS, 2020) (Fig. 3). Earth is struck by very small NEAs on a regular basis and flybys by NEAs within the Moon's orbit occur every few weeks. The surface of our moon is covered in craters from past impacts and our own planet also bears the scars of bombardment, though they are largely obscured by weathering, vegetation and the fact that the majority of Earth's surface is covered by water. Nevertheless, at the time of this writing, there are 190 concerned impact structures on Earth, many of which are larger than $20 \mathrm{~km}$ in diameter (EID, 2020).

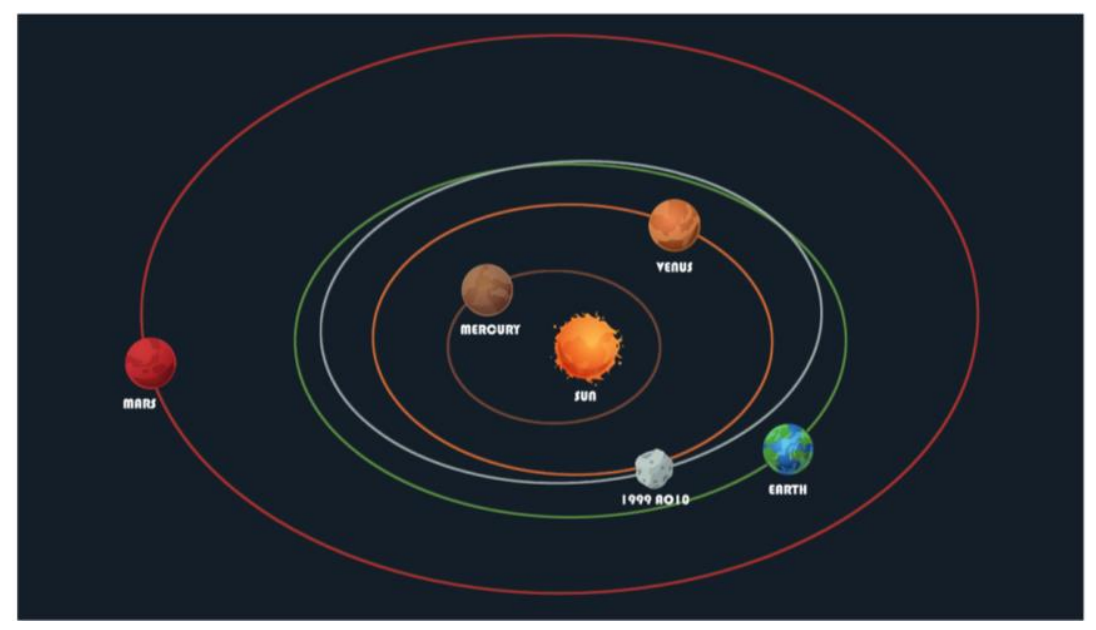

Fig. 2: Orbits of Earth and 1999 AO10 around the Sun (Credit - JPL) 


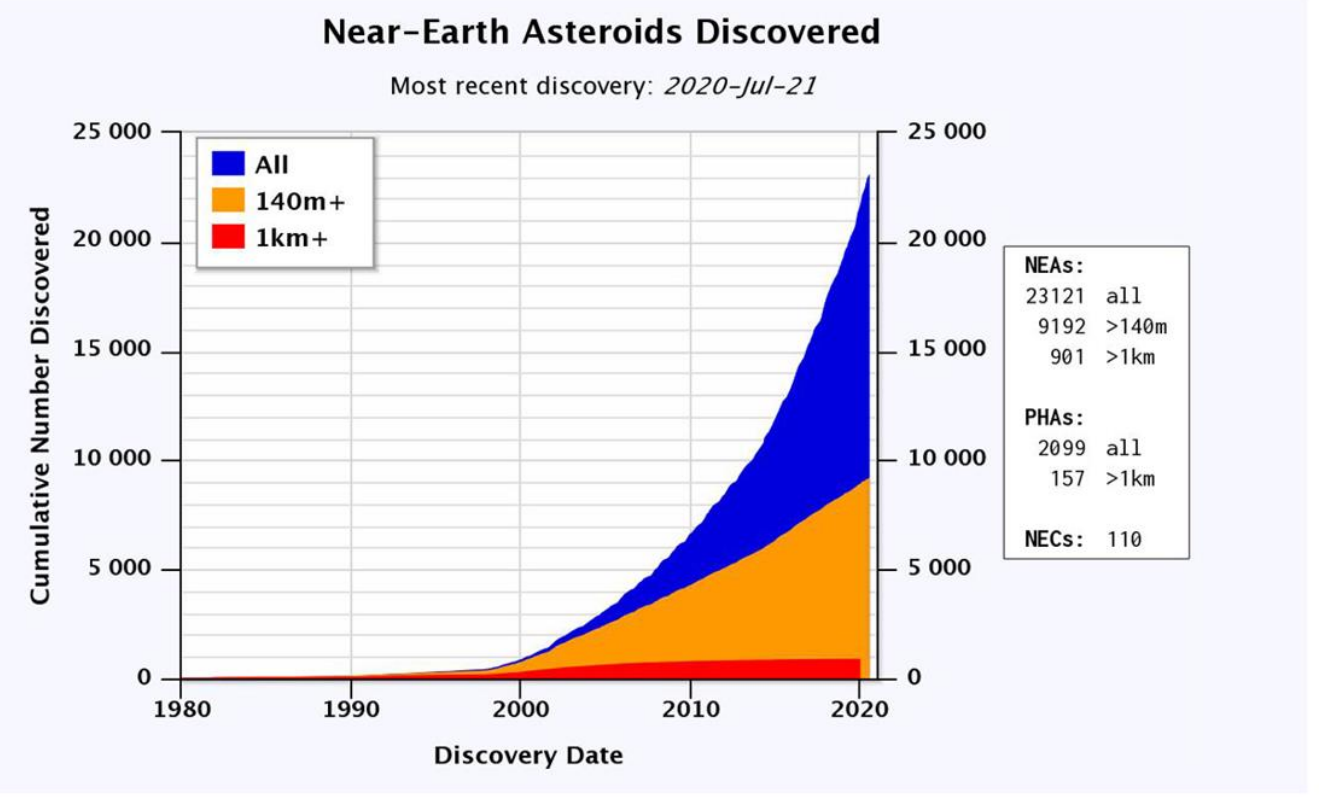

Fig. 3: Statistics of Near-Earth Asteroids discovered (Credit - JPL)

One of the biodiversity reacted in the fossil record has indicated that mass extinctions are periodic and while a direct cause has not been identified for many extinction events, we do know that the impact that created the famous Chicxulub crater in the Yucatan peninsula approximately 65 million years ago did cause the Cretaceous-Paleogene (K-Pg) extinction event, during which the dinosaurs were made extinct, along with most other species living at the time. The NEO that caused the $(\mathrm{K}-\mathrm{Pg})$ boundary extinction event is estimated to have been between 9 and $19 \mathrm{~km}$ in diameter, but the more numerous smaller NEOs can still cause considerable damage. Impacts by NEOs that are one to several $\mathrm{km}$ in diameter can cause extinction level events, while NEOs that are on the order of several hundred meters in diameters can devastate entire nations or regions. Even small NEOs that are on the order of tens of meters in size can devastate entire cities. For example, during the Tunguska event, which occurred in Siberia in the year 1908 a small NEO estimated to have been between 10 to $20 \mathrm{~m}$ in size exploded several kilometers above the ground and devastated an area the size of Washington, DC.

While our own planet and moon show ample evidence of past impact events, we have observed large scale collisions of comets and asteroids with the planet Jupiter and Mars. Impacts by NEAs are random, aperiodic events and can occur at any time with little or no warning. Our detection and characterization methods are improving (and must continue to improve), giving us the chance to have some advance warning, but NEA detection systems have yet to be built and tested. Our current and near term technology may offer the tools with which to prevent NEA impacts but we must develop and test the various proposed NEA detection systems before they can be relied upon; this includes developing proficiency with proximity operations in the vicinity of NEAs, which possess highly irregular, albeit weak, gravitational fields that have challenged the guidance and control systems of robotic spacecraft. We must also discover and characterize NEAs to inform detection system design and deployment; it is serendipitous that discovery and characterization efforts simultaneously serve the purposes of fundamental science, human exploration and planetary defense against NEA impacts.

\section{Resource Utilization}

There is a keen interest in going to asteroids in the near-earth belt. Besides being scientifically interesting and posing a threat, NEAs could be sources of valuable raw materials that could be harvested. NEAs contain useful materials such as carbon, iron, nickel, iridium, palladium, platinum, gold, magnesium, osmium, ruthenium, rhodium, water and trapped gasses such as carbon dioxide and ammonia. These resources can be utilized for a variety of purposes, including space manufacturing. It has been suggested that in late 2025s, asteroids might be used as a source of materials that may be rare or exhausted on Earth (asteroid mining) or materials for construing space habitats such as the manufacture of radiation shielding and spacecraft 
propellant, without needing to expend the tremendous energy required to launch the raw materials into space from Earth. Harnessing these resources will of course require extensive infrastructure development and a greatly scaled-up space economy. However, the first important step is to discover, explore and study NEAs so that we can survey the population, identify the available resources and develop appropriate utilization plans. Doing so will require rigorous scientific study, the ability to operate in the vicinity of NEAs and on their surfaces and the ability to modify their orbits. Thus the goal of NEA resource utilization is synergistic with the goals of solar system science, planetary defense and human exploration.

\section{Human Exploration}

While the orbital proximity of NEAs makes them a hazard to Earth, it also offers a unique opportunity since many may be accessible for human missions with very short round-trip flight time, assuming the existence of an adequate crew vehicle and heavy-lift launch capability. Human missions to NEAs would further our crucial scientific study of asteroids and simultaneously provide us with much-needed experience in true interplanetary travel prior to larger, longer duration expeditions to more distant destinations, such as Mars. Perhaps most importantly, a human mission to an NEA would be the most ambitious journey of human discovery since Apollo and would serve to reinvigorate our space program and renew public passion for space exploration.

\section{Mission Profile}

The designed profile for a crewed mission to NEA 1999 AO10 is captured in Fig. 4. Firstly, the crew module will be injected by the Earth Departure Stage (EDS) of the launch vehicle on a trajectory towards the asteroid. After completing a journey of 83 days, the crew vehicle will reach near the asteroid. Upon arrival in the vicinity of the NEA, the crew vehicle's primary thrusters will perform a maneuver to match with the NEAs orbit a small distance from NEA itself. This is the primary rendezvous maneuver along with several maneuvers performed for trajectory correction. After rendezvousing with the asteroid, the crew vehicle can station keep near the NEA or execute small trajectories to fly around it. During this period of 14 days, astronauts can perform several experiments on the surface of asteroid such as collecting the samples, spacewalk on interstellar objects, etc. After the designated stay time at the NEA has elapsed, the crew vehicles primary thrusters will perform another maneuver that will place it on a trajectory that will bring it back to Earth sometime later. However, if the natural velocity of the crew vehicle relative to Earth arising from orbital mechanics exceeds safety limits, e.g., the velocity is too high for the heat shield to tolerate, then the crew vehicle's primary thruster may perform one final maneuver to reduce its re-entry velocity accordingly.

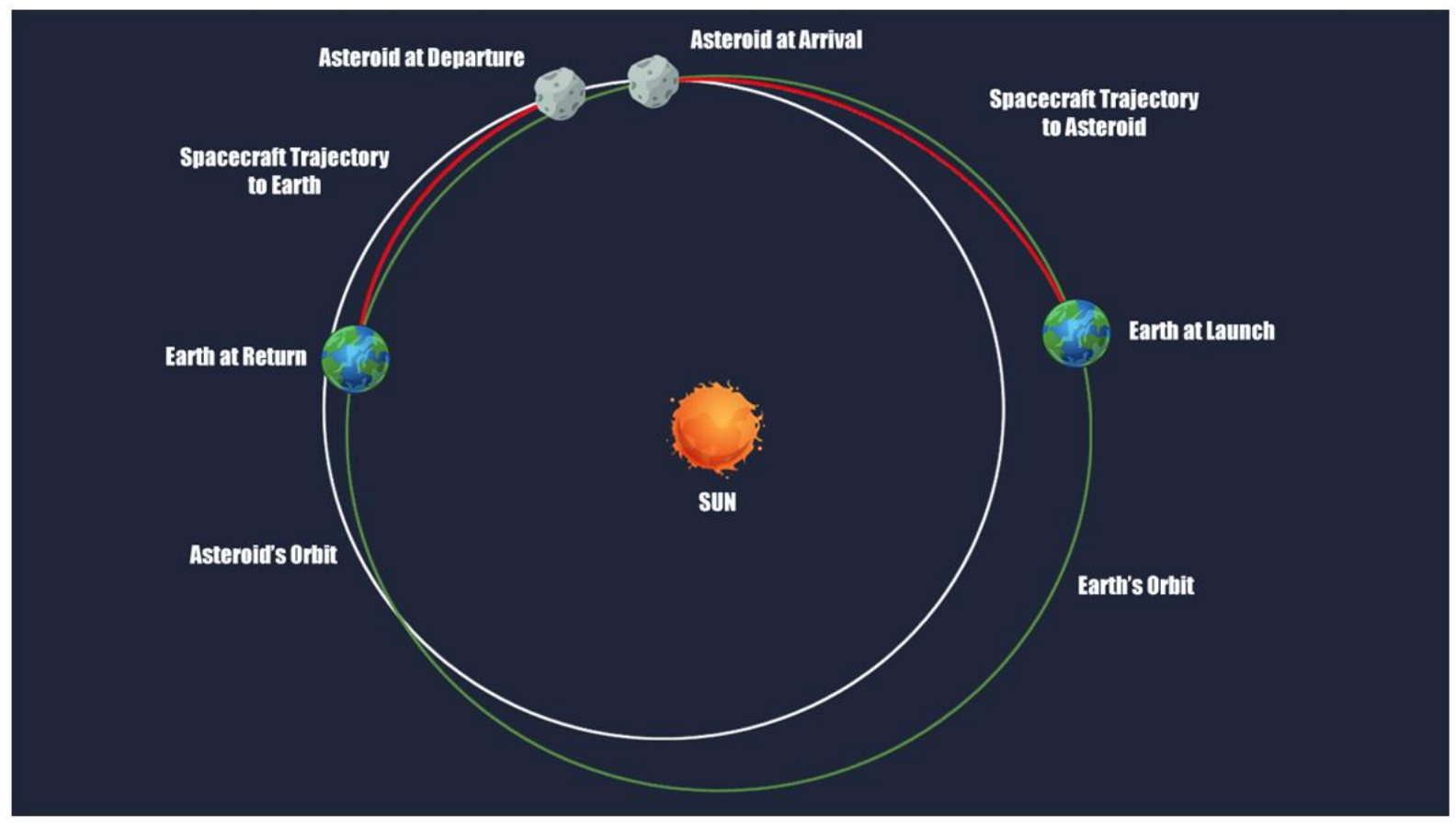

Fig. 4: Mission profile to asteroid 1999 AO10 


\section{Literature}

There are several robotic expeditions launched by space fairing nations and agencies all over the world to study asteroids in detail and return samples of their surface to the Earth if possible. Also, multiple asteroid exploration missions are currently under development. Some of these missions are mentioned below:

- In October 1989, NASA launched the Galileo Mission, which became the first spacecraft ever to encounter an asteroid when it passed Gaspra on October 29, 1991. It flew within 1,601 kilometers of the stony asteroid's center at a relative speed of about $8 \mathrm{~km} / \mathrm{s}$ while capturing its pictures and recording scientific data. The data revealed a cratered, complex, irregular body of $12.2 \mathrm{~km}$ mean diameter with a possible magnetic field. On August 28, 1993, Galileo flew within 2,400 kilometers of a second asteroid named Ida. Ida is about $31.4 \mathrm{~km}$ long and the first asteroid known to have a natural satellite (NASA, 2019a)

- Launched on February 17, 1996, Near Earth Asteroid Rendezvous-Shoemaker (NEAR Shoemaker) spacecraft was the first human-made object to orbit and the first to land on asteroid 433 Eros. NEAR's primary goal was to rendezvous with the asteroid 433 Eros and to gather data on its physical properties, mineral components, morphology, internal mass distribution and magnetic field. On the way to its primary mission, on June 27, 1997, NEAR photographed 60 percent of the asteroid 253, Mathilde, while performing a 25minute flyby from 1,200 kilometers. On February 14, 2000, NEAR orbited above the 433 Eros in an orbit of 321 x 366 kilometers. After studying for one year from different orbits NEAR finally touched down on 433 Eros on February 12, 2001. During its study, it mapped more than 70 percent of the surface, captured 160,000 images and showed no evidence of the presence of a magnetic field (NASA, 2019b)

- Deep Space 1, was launched on October 24, 1998, to demonstrate and rigorously test 12 cutting-edge technologies, including highly-efficient ion engines and autonomous navigation software. On July 29, 1999, the spacecraft flew within 26 kilometers of asteroid 9969 Braille at a velocity of about 15.5 $\mathrm{km} / \mathrm{s}$ collecting valuable data (NASA, 2019c)

- Stardust was launched on February 7, 1999, to fly through the cloud of dust that surrounds the nucleus of a comet and for the first time collect cometary material for return to Earth. On November 1, 2003, the spacecraft flew within 3,300 kilometers of asteroid Annefrank. During its flyby spacecraft collected valuable data of this $8 \mathrm{~km}$ asteroid (NASA, 2019d)

- Japan Aerospace Exploration Agency (JAXA) launched Hayabusa spacecraft on May 9, 2003, with the primary scientific objective to collect a surface sample of material from the $313 \mathrm{~m}$ diameter asteroid 25143 Itokawa and return the sample to Earth for analysis. Other scientific objectives of the mission include detailed studies of the asteroid's shape, spin state, topography, color, composition, density and interior along with its photometric and polarimetric properties. Rendezvous with the asteroid occurred on September 12, 2005, at a distance of $20 \mathrm{~km}$. Though the spacecraft was plagued with difficulties it returned samples of its surface safely to the Earth on June 12, 2010 (NASA, 2018a)

- European Space Agency (ESA) launched the Rosetta probe on March 2, 2004, to perform a detailed study of comet 67P/Churyumov-Gerasimenko. During its journey to the comet, on September 5, 2008, Rosetta flew within $800 \mathrm{~km}$ of asteroid 2867 Steins at a relative velocity of $8.6 \mathrm{~km} / \mathrm{s}$. 2867 Steins is a main E-type asteroid of about $4.6 \mathrm{~km}$. On July 10, 2010, Rosetta flew by asteroid 21 Lutetia at a distance of $3000 \mathrm{~km}$ and a speed of $15 \mathrm{~km} / \mathrm{s} .21$ Lutetia is the third-largest asteroid visited to date with a diameter of about $100 \mathrm{~km}$ (ESA, 2019)

- On September 27, 2007, NASA launched Dawn Mission to rendezvous and orbit the asteroids 4 Vesta and 1 Ceres. The scientific objectives of the mission were to characterize the asteroid's internal structure, density, shape, size, composition and mass and to return data on surface morphology, cratering and magnetism. Dawn orbited and studied 4 Vesta from July 16, 2011, to September 27, 2012 and on March 6, 2015, orbited the dwarf planet 1 Ceres. Vesta is the second-largest asteroid visited to date (NASA, 2020a)

- Launched on October 1, 2010, Chang'e 2 was the second Chinese robotic probe to orbit the Moon. On 13 December 13, 2012, Chang'e 2 flew within $3.2 \mathrm{~km}$ of the asteroid 4179 Toutatis on an extended mission capturing close-up images of the asteroid and using its instruments to acquire scientific data (NASA, 2018b)

- On December 3, 2014, JAXA launched the improved Hayabusa 2 space probe to study C-type asteroid Ryugu 162173. Hayabusa 2 along with three MINERVA-II rovers and one MASCOT lander arrived at Ryugu 162173 on June 27, 2018, to collect data and samples of it. MINERVA-II rover $1 \mathrm{~A}$ and 1B were deployed on September 21, 2018, whereas MINERVA-II rover 2 was deployed on October 3, 2019, to transmit images and data from asteroids surface. On October 3, 2018, the MASCOT rover was released from 51 meters above Ryugu's surface to collect data over one day/night $7.6 \mathrm{~h}$ rotation period. After surveying the asteroid for a year and a half Hayabusa 2 departed the asteroid on November 13, 2019, to return asteroid samples to Earth in late 2020 (NASA, 2019e)

- NASA's asteroid study and sample-return mission Origins Spectral Interpretation Resource 
Identification Security-Regolith Explorer (OSIRISRex) was launched on September 8, 2016, to study near-Earth asteroid 101955 Bennu. The primary scientific objectives are to return and analyze a sample of pristine asteroid regolith, map the global properties, chemistry and mineralogy, document the texture, morphology, volatile chemistry and spectral properties of the sample site, measure the orbit deviation caused by non-gravitational forces and characterize the integrated global properties of the asteroid for comparison with ground-based observations. On December 3, 2018, OSIRIS-Rex reached the proximity of asteroid 101955 Bennu and began to analyze its surface. It is expected to return with its sample to Earth in 2023 (NASA, 2018c)

- NASA's Double Asteroid Redirect Test (DART) mission is scheduled for launch on July 22, 2021, to evaluate the kinetic impact technique by striking an asteroid with a spacecraft at high relative velocity and observing the resulting change in orbit. It will head to the binary S-type asteroid system 65803 Didymos, consisting of a primary, Didymos A, roughly $0.8 \mathrm{~km}$ in diameter and a secondary Didymos B, roughly $160 \mathrm{~m}$ across. It will also carry the LICIA CubeSat to image the impact and its result. The impact is planned for September 30, 2022 (NASA, 2020b)

- NASA's Lucy mission is scheduled to launch on October 16, 2021, to make flyby observations of seven asteroids, one main-belt asteroid and six Trojan asteroids. The first asteroid to be encountered is 52246 Donaldjohanson, in the main asteroid belt. Donaldjohanson is a C-type asteroid with a diameter of about $4 \mathrm{~km}$. The other asteroids to be encountered represent a diverse sample of the types found in the two Trojan swarms. 3548 Eurybates is a $64 \mathrm{~km}$ diameter C-type asteroid. 15994 Polymele is a Ptype asteroid with a diameter of $21 \mathrm{~km} .11351$ Leucus is a D-type asteroid with a diameter of 34 $\mathrm{km}$ and an extremely long (447 day) rotational period. 21900 Orus is also a D-type asteroid, with a diameter of $51 \mathrm{~km}$. The last two bodies encountered are in the Jupiter trailing swarm and are a binary pair, designated 617 Patroclus-Menoetius. The primary, Patroclus, has a mean diameter of $113 \mathrm{Km}$ and Menoetius has a mean diameter of $104 \mathrm{~km}$. Both are irregularly shaped and, although they can be classified as P-type asteroids, it is possible they may actually be trapped Kuiper Belt objects, effectively comets rather than asteroids (NASA, 2020c)

- NASA's Psyche mission is designed to rendezvous with a metallic asteroid, 16 Psyche, in the main asteroid belt and study it from orbit. Psyche is scheduled to launch in August 2022. 16 Psyche is an M-type asteroid orbiting in the main asteroid belt with an estimated size of about $226 \mathrm{~km}$. The primary scientific objective of the mission is to determine whether Psyche is a core, or if it is unmelted material, determine the relative ages of regions of Psyche's surface, determine whether small metal bodies incorporate the same light elements as are expected in the Earth's high-pressure core, determine whether Psyche was formed under conditions more oxidizing or more reducing than Earth's core and characterize Psyche's topography (NASA, 2020d)

\section{Methodology}

No space mission can ever succeed without proper trajectory analysis. Maneuvering in space is not as simple as turning a steering wheel. The primary force acting on objects in space is gravity. Since gravity is such a strong force, overcoming it requires an enormous amount of energy. Therefore, spacecraft trajectory to the asteroid 1999 AO10 has to be carefully planned to minimize the expensive maneuvers required to change orbit. The first step in designing a trajectory to an asteroid is to estimate $\Delta \mathrm{V}$ and one way trip time. When making this estimate, it is helpful to ignore the Earth's sphere of influence and treat the problem as a simple transfer between two points the Earth and the asteroid. Lambert's problem is used to fit an orbit between the two positions and then Gauss' solution to Lambert's problem can be used for two-position vectors as well as a time of flight and calculate the velocity at each point which are the velocities required to form an orbit between the two bodies given the time constraint. Once the spacecraft rendezvous with the asteroid, it will stay on target. Afterward, it will depart for Earth. The return trip will proceed in a similar fashion. The next step is a calculation of $\Delta \mathrm{V}$ for impulsive transfer from Earth's orbit to the transfer orbit and from the transfer orbit to the asteroid's orbit, respectively. The process can be repeated to calculate for the return trip as well. The next step is to find the minimum total $\Delta \mathrm{V}$ for a trip. With the desired Earth-asteroid trajectory, the patched conics approach can be used to compute the required $\Delta \mathrm{V}$ accounting Earth's sphere of influence to escape Earth's orbit and put the spacecraft on a trajectory to the asteroid. This initial estimate of the required $\Delta \mathrm{V}$ from Earth to asteroid must be refined taking into account perturbations such as additional gravitational forces and solar radiation pressure.

When in the preliminary trajectory design phase, it is helpful to limit the design scope. This paper will contain analysis from such a preliminary phase and as such, will not account for perturbations such as solar radiation pressure and third-body dynamics. The spacecraft will launch in 2025's, fly to $1999 \mathrm{AO} 10$ and remain on station for specified days and return to Earth with an arrival date in 2026's. The spacecraft will start in a low earth parking orbit.

An estimated trajectory from Earth to a body in space can be computed using patched conics and a Lambert solver. The largest difference between a patched conics 
trajectory to a planet and a trajectory to an asteroid is the lack of a sphere of influence around the asteroid. Therefore, once a spacecraft leaves Earth's sphere of influence, it will be affected primarily by the Sun's gravitational field until the end of the mission, when spacecraft returns to Earth. The fundamental drivers behind the trajectory analysis of a manned mission in space are duration and destination. Mission duration in deep space is limited by radiation. Unlike a trip to Mars, where astronauts spend several months within Mars's protective atmosphere, a trip to an asteroid requires astronauts to be exposed to space radiation for the entire mission. This limits the trajectories that can be used for a trip to an asteroid. For example, a Hohmann transfer with a one-way transfer time of roughly six months is not an acceptable trajectory. In fact, six months is NASAs upper limit for total, round-trip mission time for a trip to an asteroid (Johnson, 2010).

The other fundamental driver behind trajectory analysis is the mission destination. The destination is limited by current propulsion technology. In the field of Astrodynamics, a spacecraft mass-independent value that describes propulsion requirements is $\Delta \mathrm{V}$ or the change in velocity required to make a maneuver in space. For reference, a $\Delta \mathrm{V}$ of about $11.2 \mathrm{~km} / \mathrm{s}$ is the round trip $\Delta \mathrm{V}$ for a Hohmann transfer to Mars (Wertz, 2004) and is considered achievable with current propulsion technology.

\section{Ephemeris Data}

The JPL HORIZONS Ephemeris System provides access to key solar system data and highly accurate ephemerides for all bodies present in the solar system. This contains more than 856,000 currently known asteroids including NEAs, 3598 comets, 209 natural satellites, all planets, the Sun, 163 spacecraft and several dynamical points such as Earth-Sun L1, L2, L4, L5 and system barycenters (JPL, 2020). While planetary ephemerides are available within standard published JPL ephemeris data sets, such as the DE431, ephemeris data for comets and asteroids are only available through the HORIZONS system, which offers web, email and telnet interfaces for accessing ephemeris data. Ephemeris data can be accessed in either ASCII text format or SPK format for use with JPL's NAIF SPICE toolkit. It would be faster to write down our own code in MATLAB to handle the NEA ephemerides in ASCII text format rather than learn the NAIF SPICE toolkit and make our program utilize it. Furthermore, to obtain ephemeris data files for thousands of NEAs requires an automated method to access and custom code was written to accomplish that anyhow. The solution was to first write a program capable of automatically downloading a specified list of NEA ephemeris files from the HORIZONS system via its telnet interface. These files are in ASCII text format and contain a variety of data values other than the time, position and velocity, so a small $\mathrm{C}$ program was written to extract just the time, position and velocity data and write it to a new file in a simple ASCII format that is easy to parse. The program handles all of this automatically for each NEA and the Earth. The result is a collection of properly formatted ephemeris files for each NEA and the Earth, all ready to be ingested by our accessibility analysis program. The current list of known NEAs are accessed from the NASA/JPL NEO program website. The list is displayed on the website and we then copy it into an ASCII text file and execute a program to reshape the information into the proper format for ingestion that automatically downloads all the NEA ephemeris files from the HORIZONS system. Next, a MATLAB function was written to locate the position and velocity data for a particular time value within the ephemeris table contained in a file. This function was utilized to quickly obtain the position and velocity of an NEA at a given epoch during trajectory processing after the NEA ephemeris data file was loaded into memory algorithm by the accessibility analysis program.

Our potential candidate 1999 AO10 is an Aten class NEA. It is orbiting with a semi-major axis of 0.9112417 AU with an eccentricity of 0.1122073 and a period of 0.87 years. The initial orbital elements were determined based on previous historical observations. Using Systems Tool Kit (STK) and planetary ephemeris data from JPL's small-body database, orbital elements for Earth and 1999 AO10 are propagated from September 2025 to February 2026. The classic Keplerian orbital elements for each body can be extracted resulting in an ASCII text file, looks like Table 1. The data specified for each body includes Julian Day Number, Barycentric Dynamical Time (JDTDB), Eccentricity (EC), Periapsis Distance (QR) in AU, Inclination with respect to $\mathrm{X}-\mathrm{Y}$ Plane (IN) in degrees, Longitude of Ascending Node (OM) in degrees, Argument of Perifocus (W) in degrees, Time of Periapsis (Tp) in Julian Day Number, Mean Motion (N) in degrees/day, Mean Anomaly (MA) in degrees, True Anomaly (TA) in degrees, Semi-major axis (A) in AU, Apoapsis Distance (AD) in AU and Sidereal Orbit Period (PR) in a day. From all of these provided values, constants and constraints, the dynamics of the problem can be developed.

Table 1: Sample orbital element report for asteroid 1999 AO10 from STK

\begin{tabular}{lllllll}
\hline Time $(U T C G)$ & Semi-major axis $(\mathrm{km})$ & Eccentricity & Inclination $(\mathrm{deg})$ & RAAN $(\mathrm{deg})$ & Arg. of perigee $(\mathrm{deg})$ & True anomaly $(\mathrm{deg})$ \\
\hline 18 Sept 25 & 149685835.986639 & 0.016214 & 23.438 & 0.001 & 101.405 & 253.584 \\
19 Sept 25 & 149703992.882240 & 0.016254 & 23.438 & 0.001 & 100.927 & 255.038 \\
20 Sept 25 & 149717032.245325 & 0.016314 & 23.438 & 0.001 & 100.565 & 256.377 \\
21 Sept 25 & 149724661.373412 & 0.016390 & 23.438 & 0.001 & 100.330 & 257.590 \\
22 Sept 25 & 149726834.657669 & 0.016476 & 23.438 & 0.001 & 100.225 & 258.672 \\
\hline
\end{tabular}


The relatively time invariant orbital elements (semimajor axis ' $a$ ', eccentricity ' $e$ ', inclination ' $\mathrm{i}$ ', right ascension of the ascending node ' $\Omega$ ' and argument of periapsis ' $\omega$ ') are listed below for the Earth (subscript E) and 1999 AO10 (subscript A):

$$
\begin{aligned}
& {\left[\begin{array}{l}
a_{E} \\
e_{E} \\
i_{E} \\
\Omega_{E} \\
\omega_{E}
\end{array}\right]=\left[\begin{array}{l}
149,703,993 \\
0.0163 \\
23.438^{0} \\
0.001^{0} \\
100.927^{0}
\end{array}\right]} \\
& {\left[\begin{array}{l}
a_{A} \\
e_{A} \\
i_{A} \\
\Omega_{A} \\
\omega_{A}
\end{array}\right]=\left[\begin{array}{l}
136,426,425 \\
0.1110 \\
25.296^{0} \\
355.507^{0} \\
325.026^{0}
\end{array}\right]}
\end{aligned}
$$

\section{Spacecraft Configuration}

The performance values of both the launch and crew vehicles play a crucial role in the success of any crewed exploration mission. The launch vehicle performance depends on numerous things. Also, many perturbations affect the performance of the vehicle but it can simply characterize by the amount of mass it can launch to high energy direct Earth departure trajectories. On the other hand, the crew vehicle performance is parameterized by the quantities such as dry mass, main thruster specific impulse and maximum atmospheric re-entry velocity that the vehicle can safely withstand (which is a function of the vehicle's heat shield). Therefore for this mission, SpaceX's Falcon Heavy along with Crew Dragon is chosen due to its specifications with the liberty of further changes and developments. The technical specifications for both the launch and crew vehicles are discussed below.

\section{Launch Vehicle}

Falcon Heavy is a two-stage, heavy-lift launch vehicle designed, developed and operated by SpaceX. The most powerful launcher to date is powered by Liquid Oxygen (LOX) and Rocket-grade kerosene (RP1) and can produce more than 5 million pounds of thrust at liftoff. Falcon Heavy has the highest payload carrying capacity than any other launch vehicle currently in operation. In fully expendable mode, the vehicle can inject a payload of $63,800 \mathrm{~kg}(140,660 \mathrm{lb})$ into LEO, $26,700 \mathrm{~kg}(58,860 \mathrm{lb})$ into GTO and 16,800 $\mathrm{kg}(37,040$ lb) to Mars. The partially reusable Falcon Heavy falls into a heavy-lift range of launch vehicles, capable of lifting 20 to 50 metric tons into LEO, under the classification system used by a NASA human spaceflight review panel. A fully expendable Falcon Heavy is in the super heavy-lift category with a maximum payload of 64 tons into LEO. Falcon Heavy builds on the proven, highly reliable design of Falcon 9, which minimized stage separation events and maximizes reliability.

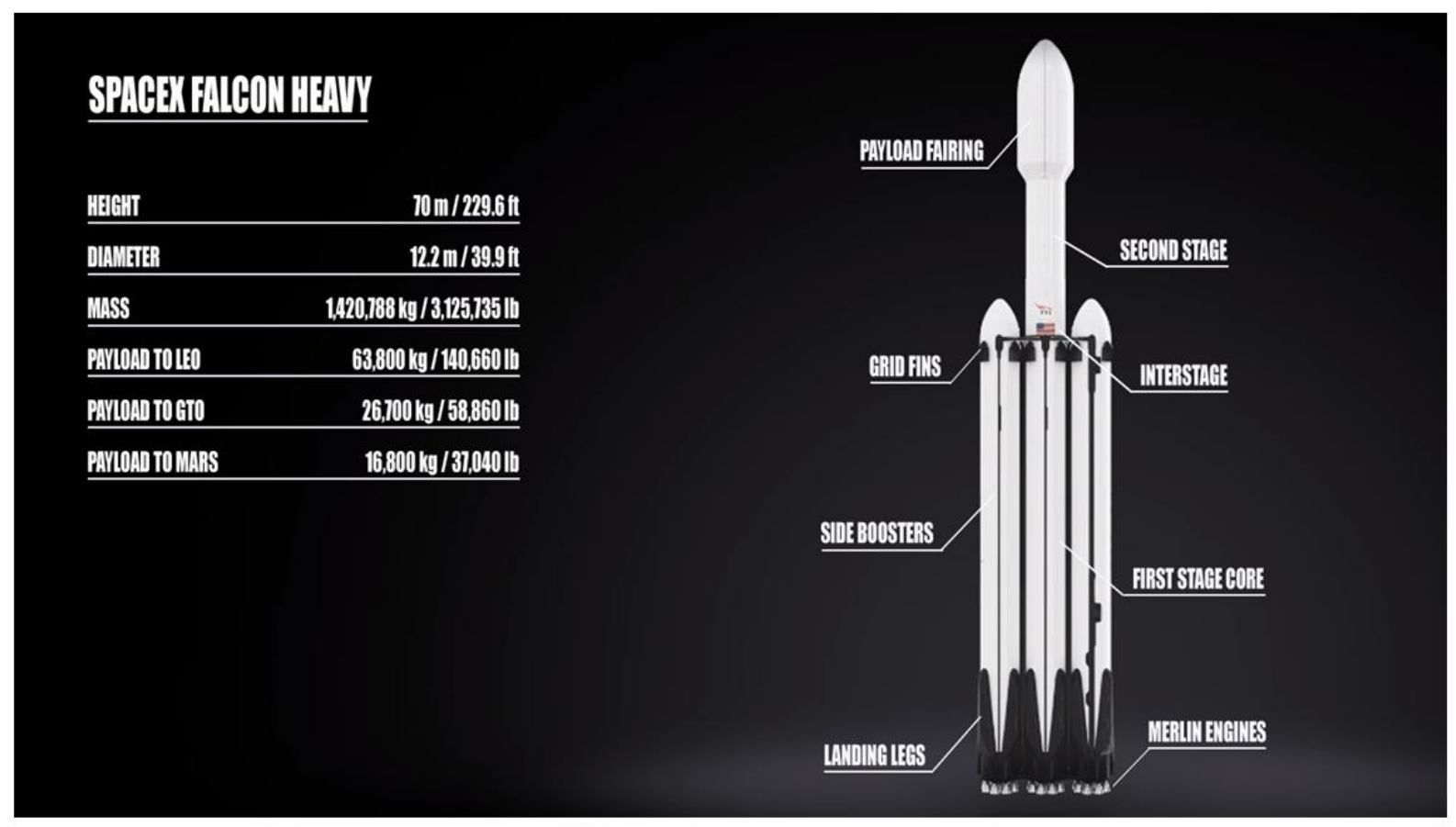

Fig. 5: Technical Specifications of Falcon Heavy (Credit - SpaceX) 
Falcon Heavy's first stage is comprised of three cores: A central core and two side boosters (the first stage of Falcon 9 is used as a side booster) with enhancements provided to strengthen the cores. Each core is equipped with nine Merlin 1D (M1D) engines which produce 845 $\mathrm{kN}(190,000 \mathrm{lb})$ of thrust per engine at sea level. Twenty-seven Merlin engines power Falcon Heavy's first stage for a total thrust of $22,819 \mathrm{kN}(5,130,000 \mathrm{lb})$ at liftoff, while thrust raised to $24,681 \mathrm{kN}(5,548,500 \mathrm{lb})$ as the craft climbs out of the atmosphere. The overall specifications of Falcon Heavy are captured in Fig. 5.

The first stage engines of the Falcon Heavy are configured in a circular pattern, with eight engines surrounding a center engine forming a structure known as Octaweb, which aimed at streamlining the manufacturing process. The side boosters are connected on the nose cone, the inter-stage and on the octaweb. With nine engines in each first-stage core, Falcon Heavy has propulsion redundancy, unlike any other heavy-lift launch system. The launch vehicle monitors each engine individually during ascent and can, if necessary, preemptively command off-nominal engines, provided the minimum injection success criteria are achievable with the remaining engines. The first stage propellant tanks of Falcon Heavy are made from aluminum-lithium alloy using friction stir welding. The center core consists of thicker tank walls and carries the booster separation system. An insulated common dome separates the LOX and RP-1 tanks and an insulated transfer tube carries LOX through the center of the RP1 tank to the engine section. The fairing is made of carbon composite material which protects the payload on their way to orbit. SpaceX is recovering fairing for reuse on future missions.

Falcon Heavy includes first stage recovery systems to allow SpaceX to return the first stage boosters to the launch site as well as recover the first stage core following landing at an Autonomous Spaceport Drone Ship barge after completion of primary mission requirements. These systems include twelve hypersonic grid fins (four on each booster), positioned at the base of inter-stage along with twelve deployable landing legs (four on each booster), stowed along the sides of each core until just prior to landing. The landing legs are made of state-of-the-art carbon fiber with an aluminum honeycomb structure. When deployed, grid fins help to control the descent of the boosters and center core through the atmosphere during re-entry. Excess propellant reserved for Falcon Heavy first stage recovery operations will be diverted for use on the primary mission objective, if required, ensuring sufficient performance margins for successful missions. Falcon Heavy's inter-stage, which connects the center core on the first and second stages, is a composite structure consisting of an aluminum honeycomb core surrounded by carbon fiber face sheet piles. The inter-stage is fixed to the forward end of the first-stage tank and holds a stage separation system. When activated stage separation occurs via reusable separation collets and a pneumatic pusher system. The second-stage tank for Falcon Heavy is a shorter version of the first-stage tank and uses most of the same material, construction, tooling and manufacturing techniques as the first-stage tank. This approach reduces manufacturing costs during vehicle production. The second stage is powered by a single Merlin Vacuum (MVac) engine, with a thrust of $981 \mathrm{kN}$ $(220,500 \mathrm{lb})$ and a fixed nozzle expansion ratio of 165:1. For added reliability of restart, the engine contains dual redundant Triethylaluminum-Triethylborane (TEA-TEB) pyrophoric igniters. In addition, the second stage contains a cold nitrogen gas $\left(\mathrm{GN}_{2}\right)$ Attitude Control System (ACS) for pointing and roll control. The $\mathrm{GN}_{2}$ ACS is more reliable and produces less contamination than a propellant-based reaction control system.

Falcon avionics feature a flight-proven, three-string, fault-tolerant architecture that has been designed to humanrating requirements. Avionics include flight computers, Global Positioning System (GPS) receivers, inertial measurement units, SpaceX designed and manufactured controllers for vehicle control (propulsion, valve, pressurization, separation and payload interfaces), a network backbone, S-band transmitters and a C-band transponder for range safety tracking. The S-band transmitters are used to transmit telemetry and video to the ground, from both the first and second stages, even after stage separation. Falcon Heavy is equipped with an autonomous flight termination system to limit the potential damage caused by a launch vehicle malfunction. The system terminates the flight of the vehicle automatically if mission rules are violated. As per the mission requirements, Falcon Heavy can be flown with a fairing or with a Dragon spacecraft. All first and second stage vehicle systems are the same in the two configurations, only the payload interface to the second stage changes between the fairing and Dragon spacecraft. Falcon Heavy architecture underwent further updates and it focused on improving the performance, reliability and life of the vehicle, as well as ensuring the vehicle's ability to meet critical government crewed and non-crewed missions. Engine performance on both stages was improved, releasing additional thrust capability. Thermal protection shielding was modified to support rapid recovery and refurbishment. Avionics designs, thrust structures and other components were upgraded for commonality, reliability and performance (SpaceX, 2020a).

\section{Crew Vehicle}

The Dragon 2 is a class of partially reusable spacecraft intended from the beginning to carry a crew of seven astronauts to and from Earth orbit and beyond. It 
is the only spacecraft currently flying that is capable of returning significant amounts of cargo to Earth and is the first private spacecraft to take humans to the International Space Station (ISS). Dragon 2 is solely designed and developed by SpaceX as the successor to the Dragon Cargo spacecraft. It has two variants: Crew Dragon, a human-rated capsule capable of carrying seven astronauts (only four seats are used for NASA missions) or a combination of crew and cargo and Cargo Dragon, an updated replacement for the original Dragon spacecraft with a payload capacity of $6,000 \mathrm{~kg}$ to the ISS. Above the seats, there is a three-screen control panel, a toilet (with privacy curtain) and the docking hatch. The spacecraft features redesigned solar arrays and a modified outer mold line compared to the original Dragon and possess new flight computers and avionics. Crew Dragon is intended to fulfill a set of requirements that will make the capsule useful to both commercial and government customers. The Dragon capsule, also known as the pressurized section, allows for the transport of people as well as environmentally sensitive cargo. The overall specifications of Dragon 2 spacecraft are shown in Fig. 6.

Dragon's trunk is the third structural element of the spacecraft, containing solar panels, heat-removal radiators and fins to provide aerodynamic stability during emergency aborts. The trunk not only carries unpressurized cargo but also supports the spacecraft during ascent. One half of the trunk is covered in solar panels which provide power to Dragon during flight and while on the station. The trunk remains attached to Dragon until shortly before re-entry into Earth's atmosphere. The deployable solar arrays have been eliminated and are now built into the trunk itself. This increases volume space, reduces the number of mechanisms on the vehicle and further increase reliability. Crew Dragon is equipped with an integrated Launch Escape System (LES) capable of accelerating the vehicle away from the rocket in an emergency at 11.8 $\mathrm{m} / \mathrm{s}^{2}$. The launch abort system is a crew safety system used to quickly separate Dragon from the launch vehicle during abort scenarios. An array of eight SuperDraco engines provides fault-tolerant propulsion for Dragon's LES. In the unlikely event of an emergency, the Crew Dragon spacecraft will use its engines to propel both the capsule and its trunk half a mile away from the launch vehicle in less than 8 seconds. Crew Dragon has eight side-mounted SuperDraco engines, clustered in redundant pairs in four engine pods, with each engine able to produce $73 \mathrm{kN}(16,400 \mathrm{lb})$ of thrust to be used for launch aborts. The Dragon spacecraft is equipped with 16 Draco thrusters used to orient the spacecraft during the mission, including apogee/perigee maneuvers, orbit adjustment and attitude control. Each Draco thruster is capable of generating $400 \mathrm{~N}(90 \mathrm{lb})$ of force in the vacuum of space. The SuperDraco engine combustion chamber is printed of Inconel, an alloy of nickel and iron, using a process of direct metal laser sintering. Engines are contained in a protective nacelle to prevent fault propagation if an engine fails.

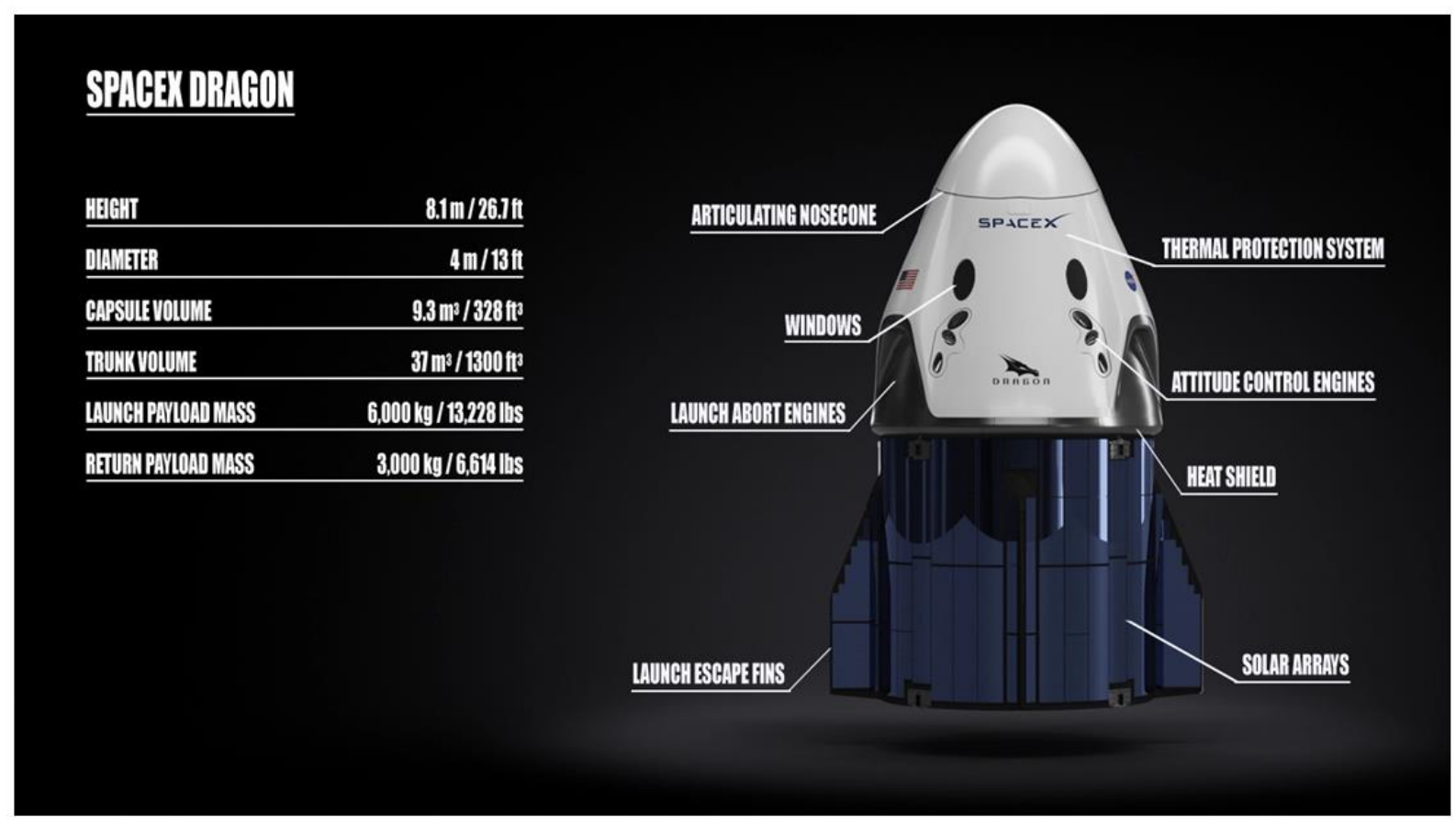

Fig. 6: Technical Specifications of Crew Dragon (Credit - SpaceX) 
Propellant and helium pressurant for both launch aborts and on-orbit maneuvering is contained in composite-carbon-overwrap titanium spherical tanks. A PICA-X heat shield protects the capsule during reentry from Lunar and Martian spaceflights, while a movable ballast sled allows more precise attitude control of the spacecraft during the atmospheric entry phase of the return to Earth and more accurate control of the landing ellipse location. A reusable nose cone protects the vessel and the docking adaptor during ascent and reentry, pivoting on a hinge to enable in-space docking and returning to the covered position for re-entry and future launches. Unlike its predecessor, the spacecraft can dock itself to the ISS instead of being berthed. It is able to perform fully autonomous rendezvous and docking with manual override ability, using the NASA Docking System (NDS). Pilots of Crew Dragon retain the ability to dock the spacecraft using manual control interfaced with a static tablet-like computer. For typical missions, Crew Dragon will remain docked to the ISS for a period of 180 days but is designed to remain on the station for up to 210 days. The spacecraft can be operated in a full vacuum. Also, the spacecraft will be able to return safely if a leak occurs of up to an equivalent orifice of $6.35 \mathrm{~mm}$ in diameter.

Crew Dragon offers each crew member a custom spacesuit fitted for them to wear during launch and reentry. This suit is primarily designed for use inside the Dragon however, in the case of a rapid cabin depressurization, the suit can protect the crew members. The suit can also provide cooling for astronauts during normal flight. The spacesuit is made from Nomex, a fire retardant fabric similar to Kevlar. The spacecraft can launch atop a Falcon 9 or Falcon Heavy rocket and returns to Earth via an ocean splashdown. Ocean landings are accomplished with two drogue parachutes to stabilize the spacecraft following re-entry and four main parachutes to further decelerate the spacecraft prior to landing. The parachute system was fully redesigned from the one used in the prior Dragon capsule, due to the need to deploy the parachutes under a variety of launch abort scenarios. As a part of NASA's Commercial Crew Program, Crew Dragon is expected to transport crews to and from the ISS. It is also expected to be used in flights by private space tourism companies to shuttle tourists to and from companies planned space station. Crew Dragon's first crewed flight with NASA astronauts occurred on May 30, 2020. This flight marked the first time a private company launched a crewed orbital spacecraft to the ISS from US soil since July 2011 (SpaceX, 2020b).

\section{Program Description}

The classic orbital elements for each body at each time can be converted to state vectors (the state vector consists of a satellite's position ' $r$ ' and velocity ' $v$ ' vectors) in a heliocentric inertial frame. First, the position and velocity vectors in the $\mathrm{PQW}$-frame are computed ( $\mathrm{P}$-axis points towards periapsis, $\mathrm{Q}$-axis points towards velocity vector, $\mathrm{W}$-axis forms a righthanded coordinate system). This is shown below, where $\mu \mathrm{s}$ is the gravitational parameter of the Sun and $v$ is the true anomaly:

$$
\begin{gathered}
P Q W_{r}=\frac{a\left(1-e^{2}\right)}{1+e \cos v}\left[\begin{array}{l}
\cos v \\
\sin v \\
0
\end{array}\right] \\
P Q W_{v}=\sqrt{\frac{\mu_{s}}{a\left(1-e^{2}\right)}}\left[\begin{array}{l}
-\sin v \\
e+\cos v \\
0
\end{array}\right]
\end{gathered}
$$

The PQW state vector can be rotated into an inertial frame using the right ascension of the ascending node, inclination and argument of periapsis combined via a rotation matrix. The rotation matrix is defined as:

$$
\left[\frac{I J K}{P Q W}\right]=\operatorname{ROT} 3(-\Omega) * \operatorname{ROT} 1(-i) * \operatorname{ROT} 3(-\omega)
$$

Here:

$$
\begin{aligned}
\text { ROT } 1 & =\left[\begin{array}{ccc}
1 & 0 & 0 \\
0 & \cos \theta & \sin \theta \\
0 & -\sin \theta & \cos \theta
\end{array}\right] \\
\text { ROT3 } & =\left[\begin{array}{ccc}
\cos \theta & \sin \theta & 0 \\
-\sin \theta & \cos \theta & 0 \\
0 & 0 & 1
\end{array}\right]
\end{aligned}
$$

ROT1 and ROT3 are the rotation matrix around the $\mathrm{X}$ and $\mathrm{Z}$ axis respectively. The rotation is shown below. Note that the vectors are in an inertial frame:

$$
r, v=\left[\frac{I J K}{P Q W}\right] P Q W_{r}, P Q W_{v}
$$

With the heliocentric inertial position vectors in hand, the trajectory design becomes an application of Lambert's problem. Gauss's solution to Lambert's problem takes two position vectors $r_{1}$ and $r_{2}$ as well as a time of flight $\Delta t$ and calculates the velocity at each point. These velocities are denoted $\mathrm{V}_{\mathrm{t} 1}$ and $\mathrm{V}_{\mathrm{t} 2}$ as a reminder that they are not the velocities of the orbiting bodies (Earth or 1999 AO10), but rather 
velocities required to form an orbit between the two bodies given the time constraint:

$$
\left[V_{t 1}, V_{t 2}\right]=\operatorname{LambertGauss}\left(r_{1}, r_{2}, \Delta t\right)
$$

The Earth's orbit and 1999 AO10's orbit, along with the transfer orbit are shown below in Fig. 7. The initial position vector $r_{1}$ is the position of the Earth at launch on September 19, 2025. The second position vector $r_{2}$ is the position of 1999 AO10 at rendezvous. The inertial reference frame is not included since Gauss's solution to Lambert's problem is driven by the position vectors relative to each other (as indicated by the difference in true anomaly $\Delta v$ or 'nu').

Once the spacecraft rendezvous with the asteroid, it will stay on target for 14 days. Afterward, it will depart for Earth. This is shown in Fig. 8, where the position $r_{3}$ is that of $1999 \mathrm{AO} 10$ at departure and the position $\mathrm{r}_{4}$ is Earth on February 21, 2026. Since the entire mission is confined to 155 days, the first transfer orbit can be computed while varying transfer time. For this orbit, $r_{1}$ will be fixed on September 19, 2025, while $r_{2}$ and $\Delta t$ will vary. The return trip will proceed in a similar fashion, varying $r_{3}$ and $\Delta t$ while fixing $r_{4}$ on February 21, 2026. Since Gauss solution to Lambert's problem calculates $\mathrm{V}_{\mathrm{t} 1}$ and $\mathrm{V}_{\mathrm{t} 2}$ and the orbital elements yielded velocity vectors $\mathrm{V}_{1}$ and $\mathrm{V}_{2}$, the scalar $\Delta \mathrm{V}_{\text {trip } 1}$ can be calculated for the first trip as follows:

$$
\begin{gathered}
\Delta V_{1}=\left|V_{t 1}-V_{1}\right| \\
\Delta V_{2}=\left|V_{2}-V_{t 2}\right| \\
\Delta V_{t r i p 1}=\Delta V_{1}+\Delta V_{2}
\end{gathered}
$$

The values of $\Delta \mathrm{V}_{1}$ and $\Delta \mathrm{V}_{2}$ represent the impulsive transfer from Earth's orbit to the transfer orbit and from the transfer orbit to the asteroid's orbit, respectively. The process can be repeated to calculate $\Delta \mathrm{V}_{\text {trip } 2}$ for the return trip as well. A sensitivity plot showing $\Delta \mathrm{V}$ vs transfer time is shown for each trip in Fig. 9. After computing all the raw accessibility data for the NEA population, a variety of post-processing steps were taken to organize and present the results. Standard plots of the trajectories were created, along with tables presenting all the key trajectory information; examples of these are presented herein with the study results. The estimated parameters of the NEAs were also computed, along with the launch and return asymptotic declination angles for the outbound and inbound trajectories. Now, as it is a manned mission, planning of the abort scenario is very necessary. Hence the post-process after the trajectory to an Asteroid is planned and the abort velocity with respect to time is calculated so that the aborted trip is feasible.

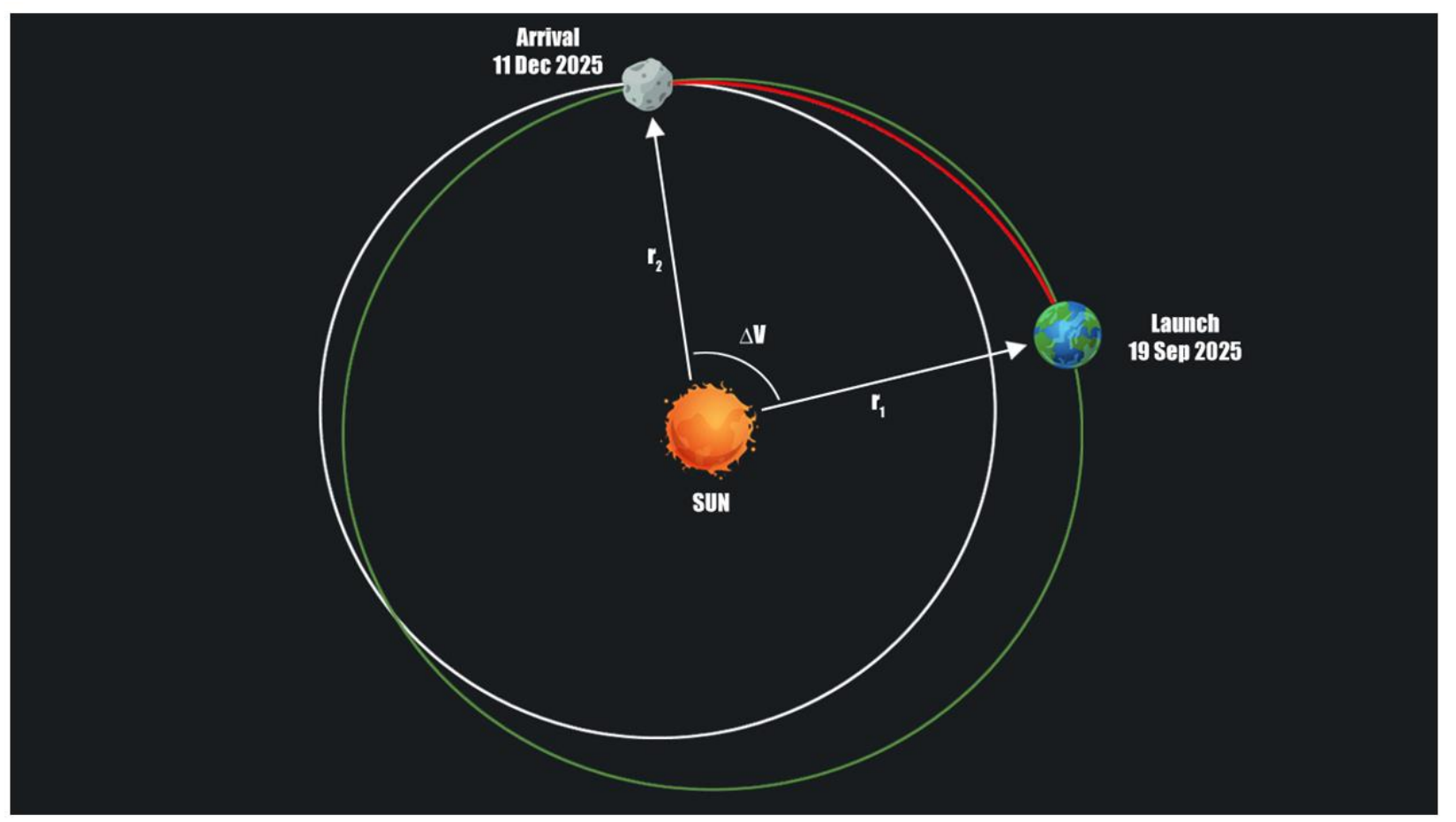

Fig. 7: Orbits of key bodies around the Sun for transfer to asteroid 1999 AO10 


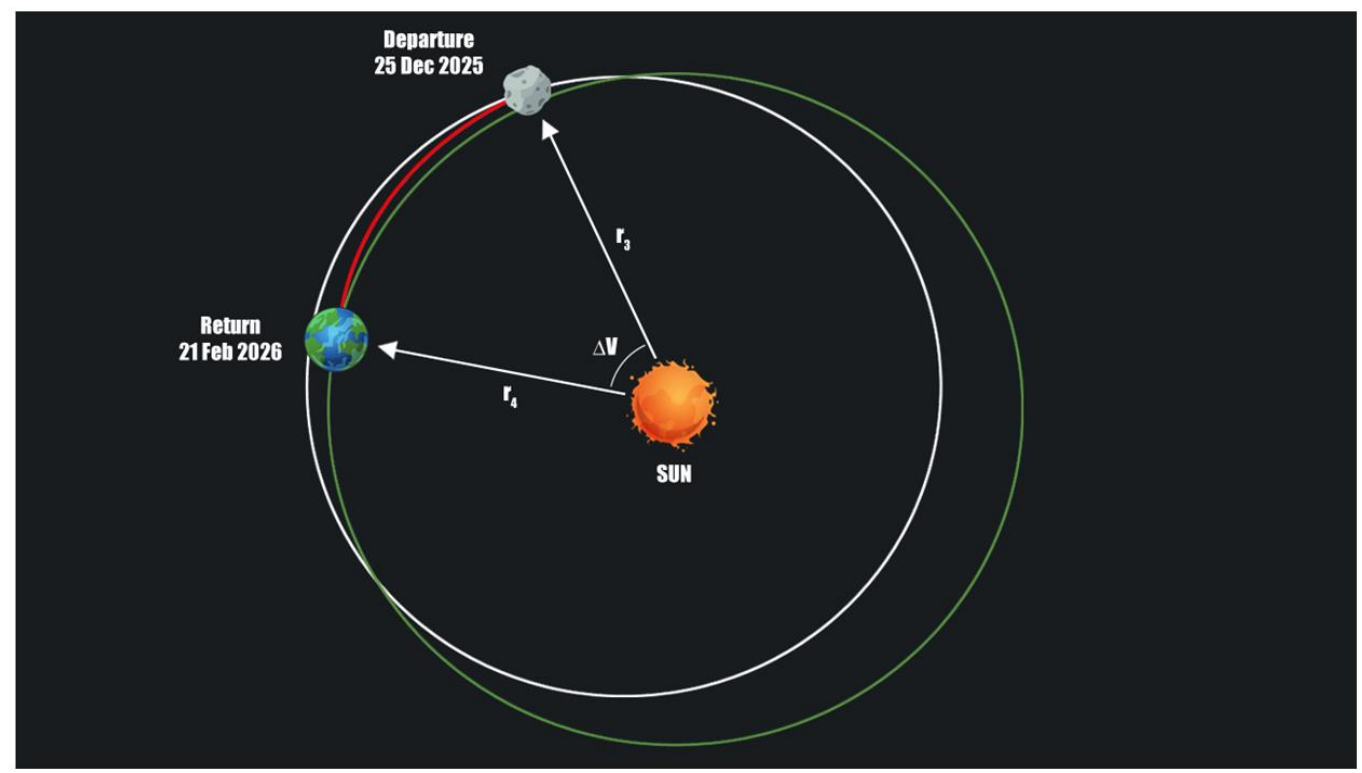

Fig. 8: Orbits of key bodies around the Sun for transfer back to Earth

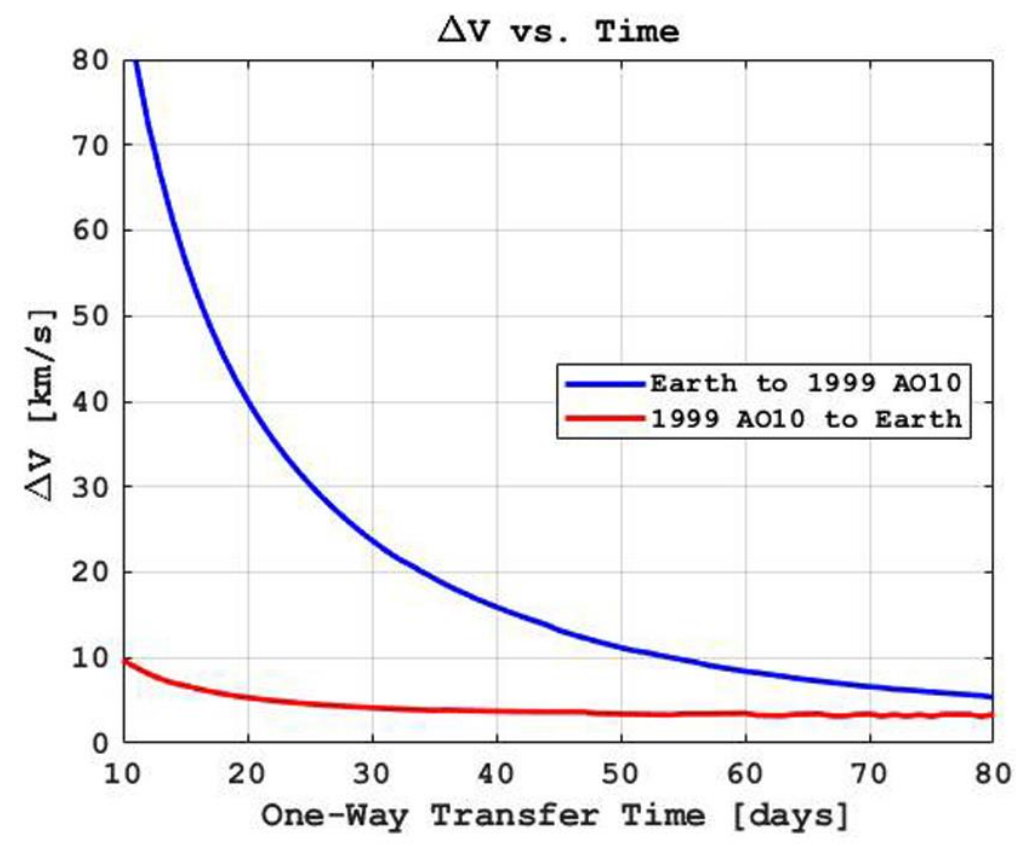

Fig. 9: Transfer time-sensitivity plot for trip to asteroid and trip back to Earth

\section{Result Analysis}

The potential candidate 1999 AO10 is a remarkable asteroid and very feasible for a human mission to deep space comparatively to all other NEAs. The optimum trajectory for the mission is calculated by performing multiple iterations using MATLAB code. The results obtained are included in the following sections. There are in total five case studies, as follows, done on the different periods to get the optimal mission trip time with minimum $\Delta \mathrm{V}$. Case I is for the years which shows the variations of the $\Delta \mathrm{V}$ for different years. Case II shows the variation in $\Delta \mathrm{V}$ according to months. Case III is done at the interval of a week. Case IV is to get a proper epoch date. Finally, Case V is done to finalize the total trip mission time in days. These five cases are mentioned below in Table 2 to 6. 
Atiksha Sharma and Santosh Kosambe / Journal of Aircraft and Spacecraft Technology 2020, Volume 4: 96.116 DOI: $10.3844 /$ jastsp.2020.96.116

Table 2: Variation of $\Delta \mathrm{V}$ w.r.t time according to years

\begin{tabular}{llllr}
\hline & & $\begin{array}{l}\text { Earth to NEA } \\
\text { transfer time }\end{array}$ & $\begin{array}{l}\text { NEA to earth } \\
\text { transfer time }\end{array}$ & $\Delta \mathrm{V} \mathrm{km/s}$ \\
\hline 2023 & Date & 124 & 17 & 867.6662 \\
2024 & 1 Jan 23-4 June 23 & 97 & 44 & 180.3804 \\
2025 & 1 Jan 24-3 June 24 & 75 & 66 & 82.9109 \\
2026 & 1 Jan 25-4 June 25 & 54 & 87 & 7.0882 \\
2027 & 1 Jan 26-4 June 26 & 63 & 78 & 88.9578 \\
\hline
\end{tabular}

Table 3: Variation of $\Delta \mathrm{V}$ w.r.t time according to months

\begin{tabular}{llllr}
\hline Year & $\begin{array}{l}\text { Month } \\
\text { (from 1 }{ }^{\text {st }} \text { day) }\end{array}$ & $\begin{array}{l}\text { Earth to NEA } \\
\text { transfer time }\end{array}$ & $\begin{array}{l}\text { NEA to Earth } \\
\text { transfer time }\end{array}$ & $\Delta$ V km/s \\
\hline $2025-2026$ & Aug-Jan & 83 & 58 & 18.0865 \\
$2025-2026$ & Sep-Feb & 82 & 59 & 10.8126 \\
$2025-2026$ & Oct-Mar & 84 & 57 & 7.6705 \\
$2025-2026$ & Nov-Apr & 85 & 56 & 6.7453 \\
$2025-2026$ & Dec-May & 70 & 71 & 6.6283 \\
2026 & Jan-Jun & 54 & 87 & 7.0882 \\
2026 & Feb-Jul & 55 & 86 & 8.9520 \\
2026 & Mar-Aug & 55 & 86 & 14.7267 \\
\hline
\end{tabular}

Table 4: Variation of $\Delta \mathrm{V}$ w.r.t time according to weeks

\begin{tabular}{llllll}
\hline Year & Month & Date & $\begin{array}{l}\text { Earth to NEA } \\
\text { transfer time }\end{array}$ & $\begin{array}{l}\text { NEA to Earth } \\
\text { transfer time }\end{array}$ & $\Delta$ km/s \\
\hline $2025-26$ & Sep-Feb & $07-08$ & 81 & 60 & 9.9297 \\
$2025-26$ & Sep-Feb & $13-15$ & 84 & 57 & 9.1690 \\
$2025-26$ & Sep-Feb & $19-21$ & 83 & 58 & 8.4940 \\
$2025-26$ & Sep-Feb & $26-28$ & 80 & 61 & 7.1237 \\
$2025-26$ & Oct-Mar & $07-11$ & 85 & 56 & 7.3984 \\
$2025-26$ & Oct-Mar & $14-18$ & 85 & 56 & 6.1773 \\
$2025-26$ & Oct-Mar & $21-25$ & 85 & 56 & 6.7926 \\
$2025-26$ & Oct-Mar & $28-01$ & 85 & 56 & \\
\hline
\end{tabular}

Table 5: Variation of $\Delta V$ w.r.t time according to dates

\begin{tabular}{llllll}
\hline Year & Month & Date & Earth to NEA transfer time & NEA to earth transfer time & $\Delta$ V km/s \\
\hline $2025-26$ & Sep-Feb & $14-16$ & 84 & 57 & 9.0561 \\
$2025-26$ & Sep-Feb & $15-17$ & 84 & 57 & 8.8440 \\
$2025-26$ & Sep-Feb & $16-18$ & 84 & 57 & 8.7280 \\
$2025-26$ & Sep-Feb & $17-19$ & 84 & 57 & 8.6189 \\
$2025-26$ & Sep-Feb & $18-20$ & 84 & 57 & 8.6711 \\
$2025-26$ & Sep-Feb & $19-21$ & 83 & 58 & 8.4940 \\
$2025-26$ & Sep-Feb & $20-22$ & 83 & 58 & 8.4296 \\
$2025-26$ & Sep-Feb & $21-23$ & 84 & 57 & 8.2443 \\
$2025-26$ & Sep-Feb & $22-24$ & 84 & 57 & 8.3875 \\
$2025-26$ & Sep-Feb & $23-25$ & 82 & 59 & 8.2814 \\
$2025-26$ & Sep-Feb & $24-26$ & 84 & 57 & 8.2800 \\
\hline
\end{tabular}

Table 6: Variation of $\Delta V$ w.r.t time according to days

\begin{tabular}{|c|c|c|c|c|c|c|}
\hline Year & Month & Date & Days & Earth to NEA transfer time & NEA to earth transfer time & $\Delta \mathrm{V} \mathrm{km} / \mathrm{s}$ \\
\hline $2025-26$ & Sep-Feb & $19-14$ & 147 & 83 & 51 & 8.6012 \\
\hline $2025-26$ & Sep-Feb & $19-15$ & 148 & 83 & 52 & 8.5241 \\
\hline $2025-26$ & Sep-Feb & $19-16$ & 149 & 83 & 53 & 8.4547 \\
\hline $2025-26$ & Sep-Feb & $19-17$ & 150 & 83 & 54 & 8.3931 \\
\hline $2025-26$ & Sep-Feb & $19-18$ & 151 & 83 & 55 & 8.3390 \\
\hline $2025-26$ & Sep-Feb & $19-19$ & 152 & 84 & 55 & 8.4241 \\
\hline $2025-26$ & Sep-Feb & $19-20$ & 153 & 83 & 57 & 8.4658 \\
\hline $2025-26$ & Sep-Feb & $19-21$ & 154 & 83 & 58 & 8.4940 \\
\hline $2025-26$ & Sep-Feb & $19-22$ & 155 & 83 & 59 & 8.4296 \\
\hline $2025-26$ & Sep-Feb & $19-23$ & 156 & 83 & 60 & 8.4216 \\
\hline $2025-26$ & Sep-Feb & $19-24$ & 157 & 81 & 63 & 8.4022 \\
\hline $2025-26$ & Sep-Feb & $19-25$ & 158 & 83 & 62 & 8.2290 \\
\hline $2025-26$ & Sep-Feb & $19-26$ & 159 & 83 & 63 & 8.2108 \\
\hline
\end{tabular}


According to the results of simulation from Table 2, it is clear that the time during late 2025 and early 2026 will give minimum $\Delta \mathrm{V}$ required for the total round trip. Accordingly, now the simulations are done for the month's gap as follows.

Considering other constraints and result from Table 3, the feasible months will be from September to December. Now, the simulations need to be done for the week's gap as follows.

In Table 4, the simulations have been done for a week's interval. By this, it is clear that the date must be around the 3rd week of September to the 1st week of February. Therefore the next simulations are done to get a proper date.

From Table 5, we came to the conclusion that the mission trip dates will be optimal from 19 September 2025 to 26 February 2026. For the total number of trip time period in terms of days, the simulations are done as below.

As per the simulation results from Table 6, it is concluded that the total trip time should be 155 days and the period should be from 19 September 2025 to 21 February 2026.

\section{MATLAB Result}

Both trips are truncated to include reasonable trip times. Note that a reasonable return trip time can be much less than that of the trip to the asteroid. This is due to the proximity of 1999 AO10 to the Earth during the return phase. The next step is to find the minimum total $\Delta \mathrm{V}$ for a trip lasting a full 155 days. This is done by iteration. The first iteration takes the initial trip time (trip 1) as fixed and finds the return trip time (trip 2) that satisfies the following equation:

$$
\begin{gathered}
\Delta t_{\text {trip } 1}+\text { HaltTime }+\Delta t_{\text {trip } 2}=\text { TotalTripTime } \\
\Delta t_{\text {trip } 1}+14 \text { days }+\Delta t_{\text {trip } 2}=155 \text { days }
\end{gathered}
$$

The next iterations move the initial trip time along the curve until a list is built of total $\Delta \mathrm{V}$, trip 1 transfer time and trip 2 transfer time. The minimum $\Delta \mathrm{V}$ is shown with the corresponding trip times in Table 7 :

$$
\begin{gathered}
\operatorname{Total} \Delta V=\Delta V_{\text {trip } 1}+\Delta V_{\text {trip } 2} \\
\operatorname{Total} \Delta V=5.0399+3.4541=8.494 \mathrm{~km} / \mathrm{s}
\end{gathered}
$$

Table 7: Minimum total mission $\Delta \mathrm{V}$

\begin{tabular}{lll}
\hline & Trip 1 & Trip 2 \\
\hline Start & 19 Sept 2025 & 25 Dec 2025 \\
Stop & 11 Dec 2025 & 21 Feb 2026 \\
Duration & 83 days & 58 days \\
$\Delta \mathrm{V}_{1}, \Delta \mathrm{V}_{3}$ & $1.5713 \mathrm{~km} / \mathrm{s}$ & $0.7525 \mathrm{~km} / \mathrm{s}$ \\
$\Delta \mathrm{V}_{2}, \Delta \mathrm{V}_{4}$ & $3.4686 \mathrm{~km} / \mathrm{s}$ & $2.7016 \mathrm{~km} / \mathrm{s}$ \\
$\Delta \mathrm{V}_{\text {trip } 1, \Delta \mathrm{V}_{\text {trip } 2}}$ & $5.0399 \mathrm{~km} / \mathrm{s}$ & $3.4541 \mathrm{~km} / \mathrm{s}$ \\
\hline
\end{tabular}

The spacecraft's position at points 1 through 4 are shown below. Note that $r_{1}$ and $r_{4}$ have large $z$ values, even though they are located at Earth. This is because STK's heliocentric frame does not define Earth's ecliptic plane as the XY-plane. Regardless, as stated above, the positions relative to each other are the important considerations when designing a trajectory to an asteroid:

$$
\begin{aligned}
& r_{1}=\left[\begin{array}{l}
149,922,771 \\
-9,700,442 \\
-4,206,531
\end{array}\right] \mathrm{km} \\
& r_{2}=\left[\begin{array}{l}
16,668,621 \\
128,470,652 \\
61,147,363
\end{array}\right] \mathrm{km} \\
& r_{3}=\left[\begin{array}{l}
-18,523,290 \\
131,728,484 \\
61,377,982
\end{array}\right] \mathrm{km} \\
& r_{4}=\left[\begin{array}{l}
-130,622,603 \\
63,728,739 \\
27,625,774
\end{array}\right] \mathrm{km}
\end{aligned}
$$

The spacecraft velocities relative to the Sun are listed below. Next to each vector is a brief description of the time during the mission that the velocity applies.

Velocity before departing Earth:

$$
V_{1}=\left[\begin{array}{l}
1.6196 \\
27.1692 \\
11.7786
\end{array}\right] \mathrm{km} / \mathrm{s}
$$

Velocity after impulsive maneuver to match transfer orbit:

$$
V_{t 1}=\left[\begin{array}{l}
0.4633 \\
26.9521 \\
12.8200
\end{array}\right] \mathrm{km} / \mathrm{s}
$$

Velocity before rendezvousing with 1999 AO10:

$$
V_{t 2}=\left[\begin{array}{l}
-31.0452 \\
3.4097 \\
1.5374
\end{array}\right] \mathrm{km} / \mathrm{s}
$$

Velocity after impulsive maneuver to match 1999 AO10's orbit velocity: 


$$
V_{2}=\left[\begin{array}{l}
-28.9600 \\
6.1658 \\
1.8329
\end{array}\right] \mathrm{km} / \mathrm{s}
$$

Velocity before departing 1999 AO10:

$$
V_{3}=\left[\begin{array}{l}
-28.9183 \\
-0.7308 \\
-1.4164
\end{array}\right] \mathrm{km} / \mathrm{s}
$$

Velocity after impulsive maneuver to match return transfer orbit:

$$
V_{t 3}=\left[\begin{array}{l}
-28.6076 \\
-1.3475 \\
-1.1174
\end{array}\right] \mathrm{km} / \mathrm{s}
$$

Velocity before rendezvousing with Earth:

$$
V_{t 4}=\left[\begin{array}{l}
-12.0520 \\
-23.1609 \\
-11.0519
\end{array}\right] \mathrm{km} / \mathrm{s}
$$

Velocity after impulsive maneuver to match Earth's orbit velocity:

$$
V_{4}=\left[\begin{array}{l}
-14.4666 \\
-24.2448 \\
-10.5103
\end{array}\right] \mathrm{km} / \mathrm{s}
$$

\section{Sphere of Influence}

With the desired Earth-asteroid trajectory in hand, the problem must now account for the Earth's sphere of influence. The first step in applying the method of patched conics is to compute the required $\Delta \mathrm{V}$ to escape Earth's orbit and put the spacecraft on a trajectory towards 1999 AO10. A pictorial representation of an escape trajectory is shown in Fig. 10. The spacecraft begins in Low Earth Orbit (LEO) with a velocity relative to the Earth as $\mathrm{V}_{\mathrm{SC} / \mathrm{E}}$. At the periapsis of the escape hyperbolic orbit, the spacecraft increases its velocity to $\mathrm{V}_{\mathrm{P} / \mathrm{E}}$ such that the spacecraft's velocity is $\mathrm{V}_{\infty / \mathrm{E}}$ when it leaves Earth's sphere of influence.

From the analysis above, the required velocity of the spacecraft relative to the Sun $V_{t 1}$ is known as well as the speed of the Earth relative to the Sun $V_{E}=V_{1}$. Therefore, the spacecraft's velocity must be increased by the hyperbolic excess velocity relative to the $\operatorname{Sun} \mathrm{V}_{\infty / \mathrm{s}}$, shown below (note that $\mathrm{V}_{\infty / \mathrm{s}}$ is the vector form of $\Delta \mathrm{V}_{1}$ ):

$$
\begin{gathered}
V_{\infty / s}=V_{t 1}-V_{E} \\
V_{\infty / s}=\left[\begin{array}{l}
0.4633 \\
26.9521 \\
12.8200
\end{array}\right]-\left[\begin{array}{l}
1.6196 \\
27.1692 \\
11.7786
\end{array}\right]=\left[\begin{array}{l}
-1.1563 \\
-0.2171 \\
1.0414
\end{array}\right] \mathrm{km} / \mathrm{s}
\end{gathered}
$$

The spacecraft trajectory from Earth to 1999 AO10 begins in a circular LEO, with an orbital radius of $\mathrm{r}_{\mathrm{SC}}=$ $6678 \mathrm{~km}$ (300 km altitude) and an orbital speed relative to the Earth $\mathrm{V}_{\mathrm{SC} / \mathrm{E}}$, where $\mu_{\mathrm{E}}$ is the gravitational parameter of the Earth:

$$
V_{s c / E}=\sqrt{\mu_{E} / r_{s c}}=7.7258 \mathrm{~km} / \mathrm{s}
$$

Since the transfer from Earth to 1999 AO10 requires an inclination change, computing the $\Delta \mathrm{V}$ to escape Earth requires an additional assumption: the LEO of the spacecraft must be in the same orbital plane as the transfer orbit (relative to the Sun) at the time of the escape burn. This assumption allows the hyperbolic escape trajectory to be converted to an Earth-centered co-ordinate system without worrying about a coordinate transformation $\left(\mathrm{V}_{\infty / \mathrm{E}}=\mathrm{V}_{\infty / \mathrm{s}}\right)$.

The next step is finding the speed at the hyperbolic orbit's periapsis, calculated below. Because the staging orbit is circular, the radius at hyperbolic periapsis is simply $\mathrm{r}_{\mathrm{SC} / \mathrm{E}}$ :

$$
V_{p / E}=\sqrt{V_{\infty / E}^{2}+\frac{2 \mu_{E}}{r_{p / E}}}=\sqrt{V_{\infty / E}^{2}+\frac{2 \mu_{E}}{r_{s c / E}}}=10.9977 \mathrm{~km} / \mathrm{s}
$$

Finally, escape $\Delta \mathrm{V}_{\text {esc }}$ can be calculated

$$
\Delta V_{e s c}=V_{p / E}-V_{s c / E}=10.997-7.7258=3.2718 \mathrm{~km} / \mathrm{s}
$$

This process can be repeated for the return trip as well. When the spacecraft returns to Earth, it must perform a burn with a change in the speed of $\Delta \mathrm{V}_{\text {cap }}$ to return to a $300 \mathrm{~km}$ LEO. The escape $\Delta \mathrm{V}$ and capture $\Delta \mathrm{V}$ replace the original $\Delta \mathrm{V}_{1}$ and $\Delta \mathrm{V}_{4}$ since these burns must now account for the Earth's gravitational field. The resulting trip $\Delta \mathrm{V}$ is shown in Table 8 :

Table 8: Mission $\Delta \mathrm{V}$ including adjustment for patched conics

\begin{tabular}{ll}
\hline Stage & Value \\
\hline$\Delta V_{\text {esc }}$ & $3.2718 \mathrm{~km} / \mathrm{s}$ \\
$\Delta V_{2}$ & $3.4686 \mathrm{~km} / \mathrm{s}$ \\
$\Delta V_{3}$ & $0.7525 \mathrm{~km} / \mathrm{s}$ \\
$\Delta V_{\text {cap }}$ & $3.3231 \mathrm{~km} / \mathrm{s}$ \\
\hline
\end{tabular}




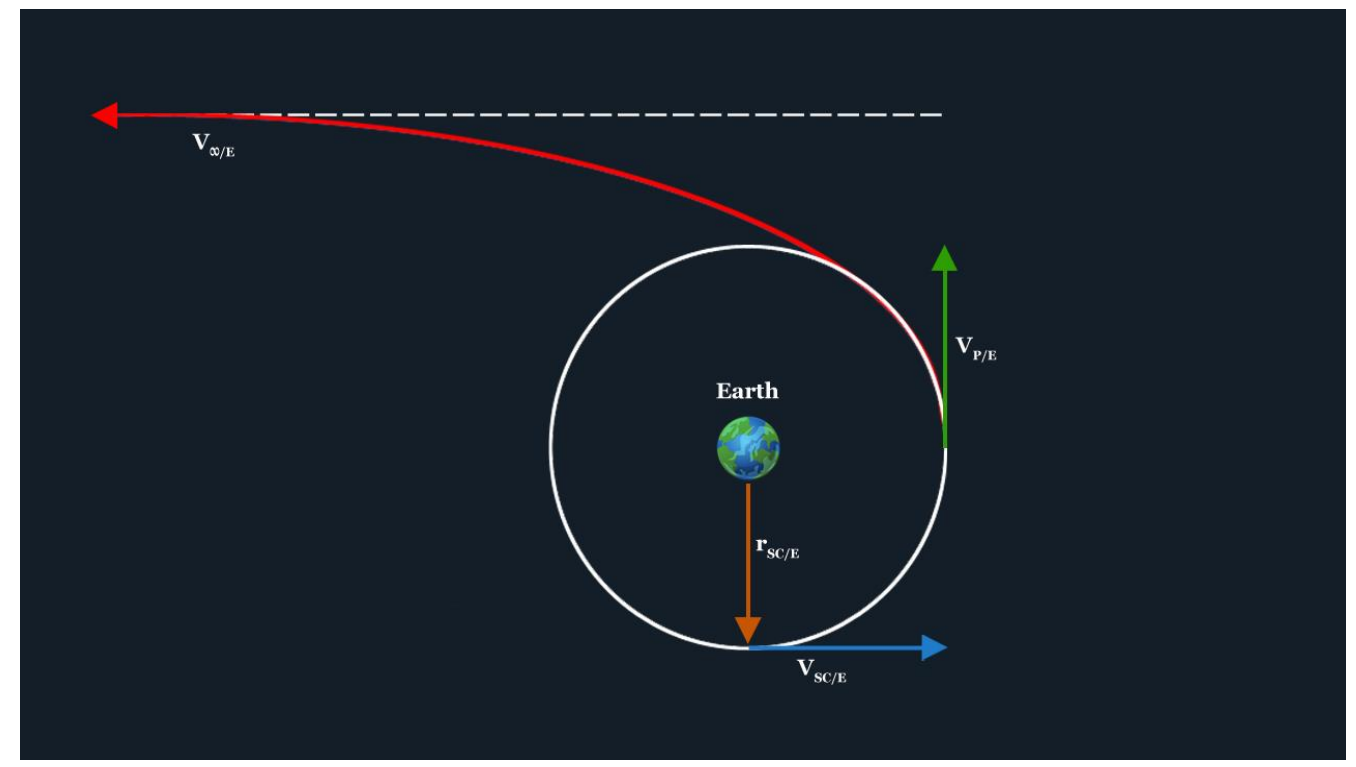

Fig. 10: Escape Trajectory for spacecraft leaving Earth

Total $\Delta V=\Delta V_{e s c}+\Delta V_{2}+\Delta V_{3}+\Delta V_{\text {cap }}=10.8160 \mathrm{~km} / \mathrm{s}$

Note that the mission $\Delta \mathrm{V}$ was increased by 2.3221 $\mathrm{km} / \mathrm{s}$ due to the necessity to escape Earth's gravity. Even with this increased $\Delta \mathrm{V}$, the propulsion requirements for a trip to 1999 AO10 are less than a trip to Mars. A simple Hohmann transfer to Mars requires $\Delta \mathrm{V}=11.2 \mathrm{~km} / \mathrm{s}$, which does not account for the energy required to escape Earth's or Mars gravitational field.

Now that an initial estimate of the required $\Delta \mathrm{V}$ for a manned trip from Earth to 1999 AO10 has been made, the bulk of the mission's Astrodynamics work has been completed. Of course, the $\Delta \mathrm{V}$ must be refined, taking into account perturbations such as additional gravitational forces and solar radiation pressure. These refinements are outside the scope of this paper. There is, however, another Astrodynamics problem that must be considered.

\section{Abort Scenario}

Since the mission under consideration is a crewed mission, certain safety factors have to be evaluated. One such factor is creating a return trajectory in case of catastrophic failure during the trip to 1999 AO10. This extension is limited to abort scenarios during the outbound trip, though it can be applied to the return trip as well. Recall that the total trip $\Delta \mathrm{V}$ is approximately $10.8 \mathrm{~km} / \mathrm{s}$. Since an abort during the outbound trip implies the spacecraft will no longer rendezvous with $1999 \mathrm{AO} 10, \Delta \mathrm{V}_{2}$ and $\Delta \mathrm{V}_{3}$ from Table 8 will no longer apply. Instead, the intermediate $\Delta \mathrm{V}$ values will be replaced by a single abort $\Delta \mathrm{V}_{\mathrm{abt}}$, designed to direct the spacecraft back to Earth. From Table $8, \Delta \mathrm{V}_{2}$ and $\Delta \mathrm{V}_{3}$ sum to approximately $4.2 \mathrm{~km} / \mathrm{s}$. Since $\Delta \mathrm{V}$ translates into a mass, which is expensive to launch, an abort $\Delta \mathrm{V}_{\mathrm{abt}}$ must be limited. For the following analysis, $\Delta \mathrm{V}_{\text {abt }}$ is limited to $6 \mathrm{~km} / \mathrm{s}$, a $30 \%$ increase over the designed mission intermediate $\Delta \mathrm{V}$. Of course, during the design of the spacecraft, this $\Delta \mathrm{V}$ limit may have to increase in the case of an emergency. However, for this mission, a reasonable fixed value will work. With the transfer state vectors from designing a trajectory, the transfer orbit from Earth to 1999 AO10 can be propagated. This means that the spacecraft position and velocity at each point along the outbound trajectory are known. An abort sequence can be initiated at each point during the 83 day trip to 1999 AO10. Since a viable return route is unknown, each return trajectory has two variables, $\Delta \mathrm{V}_{\mathrm{abt}}$ and transfer time. These values can be calculated using the same iterative process described in designing a trajectory. The result is a function of $\Delta \mathrm{V}_{\text {abt }}$ vs return transfer time for each day from day 1 to day 83, as shown in Fig. 11 and 12. Figures 11 and 12 show a couple of interesting patterns. First, as the mission abort day increases from 1 to 71 days, the minimum required $\Delta \mathrm{V}$ decreases. At day 71, an inflection occurs and the minimum $\Delta \mathrm{V}$ begins to increase up to day 83 . Second, a viable abort sequence only exists for a relatively small window. Figure 11 shows that, given the $6 \mathrm{~km} / \mathrm{s}$ limit, an abort sequence only exists beginning with day 63 .

If an emergency occurs before that, the crew will have to wait at least until day 63 to initiate an abort sequence. While shifting trajectories on day 63 will return the crew to Earth, the return time is on the order of 
60 days. That means the total mission time is about 120 days. The object of an abort sequence is to minimize the total mission time. If an abort scenario is required, the crew has limited options. So if an emergency occurs before day 71, the crew will have to wait until the 71st day to turn the spacecraft around as shown in Fig. 11. If an abort maneuver is performed on day 71 , the return time is only about 13 days, shortening the mission duration to 84 days. Figure 12 shows after the 71st day of the mission, the return time for a $\Delta \mathrm{V}_{\text {abt }}$ less than 6 $\mathrm{km} / \mathrm{s}$ begins to increase. The longer the crew waits, the longer the trip back will be. Therefore, if an emergency occurs after day 71, the crew's best option is to head back to Earth immediately. Abort scenarios exist while within Earth's sphere of influence. One such scenario involves using the moon's gravity to sling the spacecraft back to Earth. While such a case was not considered in this paper, it is an important consideration during the next phases of mission design. The result of a moonabort scenario would be a window early in the mission when the crew can quickly return to Earth. Unfortunately, once the spacecraft leaves the gravity field of the Earth, such a scenario no longer exists.

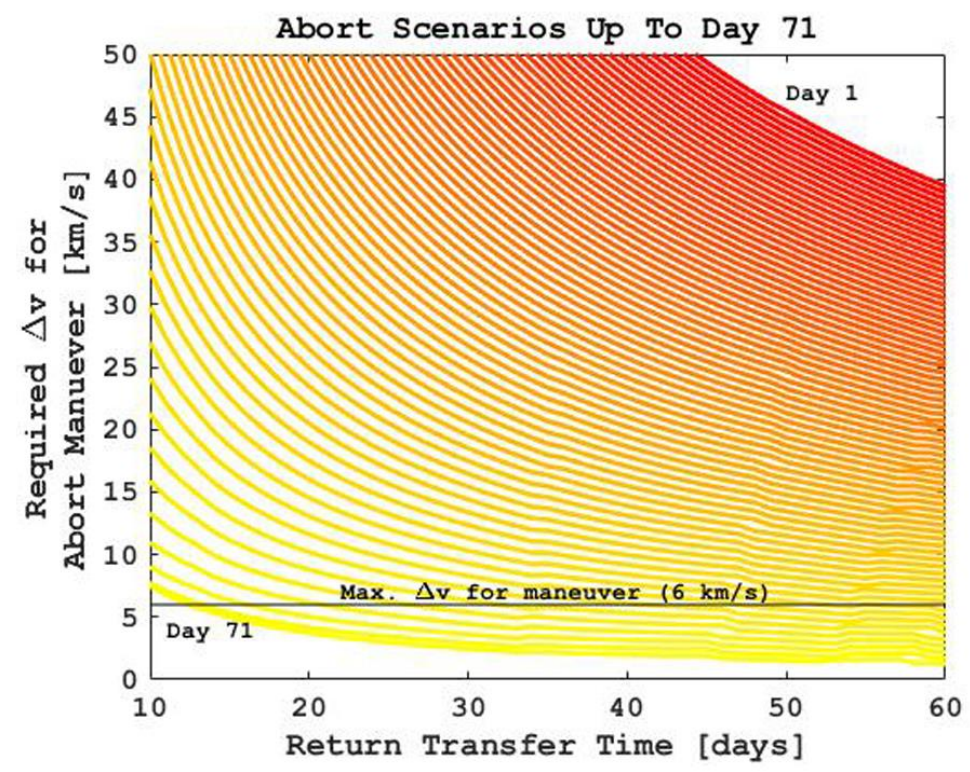

Fig. 11: Required $\Delta \mathrm{V}$ to abort mission during between days 1 and 71

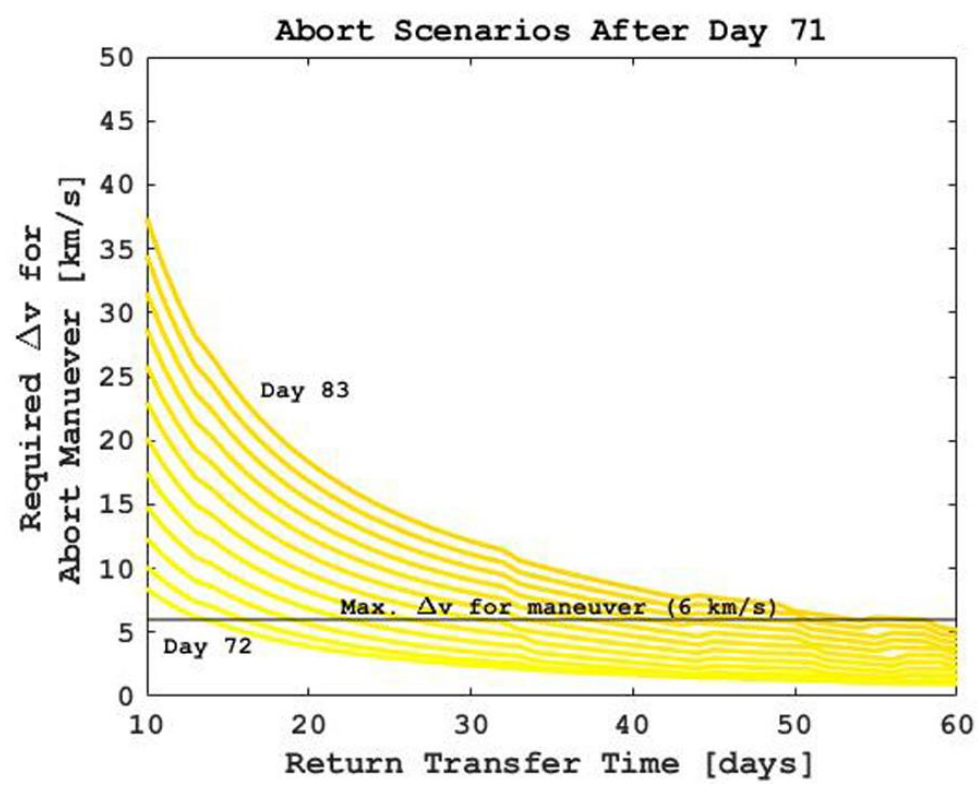

Fig. 12: Required $\Delta \mathrm{V}$ to abort mission during between days 72 and 83 


\section{Conclusion}

A manned mission to an asteroid is more complex than a manned mission to Mars. Designing the trajectory requires the creative use of Lambert's problem and some iterating to arrive at an optimal path. However, a preliminary trajectory is feasible to design using basic Astrodynamics. This initial trajectory estimate revealed a total trip $\Delta \mathrm{V}$ of 10.8 $\mathrm{km} / \mathrm{s}$, which is within the range of feasibility. Space agencies may choose to send humans on an unprecedented deep-space journey to an NEA 1999 AO10 in the year 2025 and to inform this effort we have developed a robust, flexible and highly capable algorithm that can identify trajectories to NEAs accessible for human exploration. Today the asteroid is a huge topic of discussion worldwide. Many astronomers and scientists are heading there steps towards the asteroids. Numerous asteroids have good physical properties which can be helpful for earthly purposes. The main advantage of this paper is the MATLAB code generated for designing the trajectory with minimum $\Delta \mathrm{V}$ and time of flight to any targeted asteroid. The paper is to propose a proper plan to reach targeted asteroid 1999 AO10 for studying and gaining the knowledge of the physics of an asteroid and also to extract its usefulness. The future plan is to implement the proposed plan by incorporating the perturbations to the actual mission. There are many ongoing Asteroids projects in various space organizations like the Soviet Union, ESA, JAXA and NASA. They are ready to implement their projects from 2025 to 2026. ISRO is also working in this field and planning to implement the Asteroid's project in late 2025. Trajectory optimization is an exciting and valuable field, the importance of which is growing exponentially. As technology advances, mankind's ability to improve upon the foundations of the past progresses as well.

\section{Acknowledgement}

The authors would like to thank unknown reviewers for their thoughtful suggestions and comments.

\section{Author's Contributions}

All authors equally contributed in this work.

\section{Ethics}

This article is original and contains unpublished material. The corresponding author confirms that all of the other authors have read and approved the manuscript and no ethical issues involved.

\section{References}

CNEOS. (2020). Center for Near Earth Object Studies, Jet Propulsion Laboratory (JPL), Discovery Statistics. https://cneos.jpl.nasa.gov/stats/totals.html

EID. (2020). Earth Impact Database. http://www.passc.net/EarthImpactDatabase/New\%2 0website_05-2018/Index.html

ESA. (2019). Rosetta overview, European Space Agency, Science and Exploration. https://www.esa.int/Science_Exploration/Space_Sci ence/Rosetta_overview

Johnson, L. (2010). Opportunities for Near Earth Object Exploration. Exploration of Near Earth Objects (NEO) Objectives Workshop. Mayflower Renaissance Hotel, Washington.

JPL. (2020). Solar System Dynamics (SSD), Jet Propulsion Laboratory (JPL), NASA. https://ssd.jpl.nasa.gov/?horizons_doc\#intro

MPC. (2020). The International Astronomical Union, Minor Planet Center. https://minorplanetcenter.net/

NASA. (2018a). Hayabusa, National Aeronautics and Space Administration (NASA) Science, Solar System Exploration. https://solarsystem.nasa.gov/missions/hayabusa/indepth/

NASA. (2018b). Chang'e 2, National Aeronautics and Space Administration (NASA) Science, Solar System Exploration. https://solarsystem.nasa.gov/missions/change-2/indepth/

NASA. (2018c). OSIRIS-REx, National Aeronautics and Space Administration (NASA) Science, Solar System Exploration. https://solarsystem.nasa.gov/missions/osiris-rex/indepth/

NASA. (2019a). Galileo, National Aeronautics and Space Administration (NASA) Science, Solar System Exploration. https://solarsystem.nasa.gov/missions/galileo/overvi ew/

NASA. (2019b). NEAR Shoemaker, National Aeronautics and Space Administration (NASA) Science, Solar System Exploration. https://solarsystem.nasa.gov/missions/nearshoemaker/in-depth/

NASA. (2019c). Deep Space 1, National Aeronautics and Space Administration (NASA) Science, Solar System Exploration. https://solarsystem.nasa.gov/missions/deep-space1/in-depth/

NASA. (2019d). Stardust/Stardust NExT, National Aeronautics and Space Administration (NASA) Science, Solar System Exploration. https://solarsystem.nasa.gov/missions/stardust/indepth/ 
NASA. (2019e). Hayabusa 2, National Aeronautics and Space Administration (NASA) Science, Solar System Exploration. https://solarsystem.nasa.gov/missions/hayabusa2/in-depth/

NASA. (2020a). DART, National Aeronautics and Space Administration (NASA), Science, Solar System Exploration.

https://dart.jhuapl.edu/Mission/index.php

NASA. (2020b). Dawn, National Aeronautics and Space Administration (NASA) Science, Solar System Exploration.

https://solarsystem.nasa.gov/missions/dawn/overvie $\mathrm{w} /$
NASA. (2020c). Lucy, National Aeronautics and Space Administration (NASA), Science, Solar System Exploration. http://lucy.swri.edu//

NASA. (2020d). Psyche, National Aeronautics and Space Administration (NASA) Science, Solar System Exploration. https://psyche.asu.edu/

SpaceX. (2020a). Falcon Heavy. https://www.spacex.com/vehicles/falcon-heavy/

SpaceX (2020b). Dragon. https://www.spacex.com/vehicles/dragon/

Wertz, J.R. (2004). Interplanetary round trip mission design." AIAA lunchtime seminar. El Segundo. 